\title{
Lower algebraic $K$-theory of hyperbolic 3-simplex reflection groups
}

\author{
Jean-François Lafont and Ivonne J. Ortiz
}

\begin{abstract}
A hyperbolic 3-simplex reflection group is a Coxeter group arising as a lattice in $O^{+}(3,1)$, with fundamental domain a geodesic simplex in $\mathbb{H}^{3}$ (possibly with some ideal vertices). The classification of these groups is known, and there are exactly 9 cocompact examples, and 23 non-cocompact examples. We provide a complete computation of the lower algebraic $K$-theory of the integral group ring of all the hyperbolic 3-simplex reflection groups.
\end{abstract}

Mathematics Subject Classification (2000). 19A31, 19B28, 19D35, 18F25, 16E20.

Keywords. Classifying spaces, lower algebraic $K$-theory, Coxeter groups, hyperbolic manifold, Farrell-Jones isomorphism conjecture, relative assembly map, Waldhausen Nil-groups, Farrell Nil-groups, Bass Nil-groups.

\section{Introduction}

In this paper, we proceed to give a complete computation of the lower algebraic $K$-theory of the integral group ring of all the hyperbolic 3 -simplex reflection groups.

We now proceed to outline the main steps of our approach. Since the groups $\Gamma$ we are considering are lattices inside $O^{+}(3,1)$, fundamental results of Farrell and Jones [FJ93] imply that the lower algebraic $K$-theory of the integral group ring $\mathbb{Z} \Gamma$ can be computed by calculating $H_{n}^{\Gamma}\left(E_{\mathcal{V} C}(\Gamma) ; \mathbb{K} \mathbb{Z}^{-\infty}\right)$, a specific generalized equivariant homology theory for a model for the classifying space $E_{\mathcal{V C}}(\Gamma)$ of $\Gamma$ with isotropy in the family $\mathcal{V} C$ of virtually cyclic subgroups of $\Gamma$.

After introducing the groups we are interested in (see Section 2), we then combine results from our previous paper [LO07] with a recent construction of Lück and Weiermann [LW] to obtain the following explicit formula for the homology group above:

$$
K_{n}(\mathbb{Z} \Gamma) \cong H_{n}^{\Gamma}\left(E_{\mathcal{F} \mathcal{I N}}(\Gamma) ; \mathbb{K} \mathbb{Z}^{-\infty}\right) \oplus \bigoplus_{i=1}^{k} H_{n}^{V_{i}}\left(E_{\mathcal{F} \mathcal{I N}}\left(V_{i}\right) \rightarrow *\right)
$$

In the formula above, $E_{\mathscr{F} \mathcal{I N}}(\Gamma)$ is a model for the classifying space for proper actions (i.e., with isotropy in the family $\mathcal{F} \mathcal{I} \mathcal{N}$ of finite subgroups), the collection $\left\{V_{i}\right\}_{i=1}^{k}$ are 
a finite collection of virtually cyclic subgroups with specific geometric properties, and $H_{n}^{V_{i}}\left(E_{\mathscr{F} \mathcal{I N}}\left(V_{i}\right) \rightarrow *\right)$ are cokernels of certain relative assembly maps. This explicit formula is obtained in Section 3.

In view of this explicit formula, our computation reduces to being able to

(1) identify, for each of our groups, the corresponding collection $\left\{V_{i}\right\}$ of virtually cyclic subgroups (done in Section 4),

(2) to calculate the cokernels of the corresponding relative assembly maps (done in Section 6), and

(3) to calculate the homology groups $H_{n}^{\Gamma}\left(E_{\mathcal{F} \mathcal{I N}}(\Gamma) ; \mathbb{K} \mathbb{Z}^{-\infty}\right)$.

For the computation of the homology groups, we note that Quinn [Qu82] has developed a spectral sequence for computing the groups $H_{n}^{\Gamma}\left(E_{\mathcal{F} \mathcal{I N}}(\Gamma) ; \mathbb{K}^{-\infty}\right)$. The $E^{2}$-terms in the spectral sequence can be computed in terms of the lower algebraic $K$-theory of the stabilizers of cells in a CW-model for the classifying space $E_{\mathscr{F} I \mathcal{N}}(\Gamma)$.

In Section 5, we proceed to give, for each of the finite subgroups appearing as a cell stabilizer, a computation of the lower algebraic $K$-theory. We return to the spectral sequence computation in Section 7, where we analyze some of the maps appearing in the computation of the $E^{2}$-terms for the Quinn spectral sequence. In all 32 cases, the spectral sequence collapses at the $E^{2}$ stage, allowing us to complete the computations. The reader who is merely interested in knowing the results of the computations is invited to consult Table 7 (for the non-uniform lattices) and Table 8 (for the uniform lattices). Finally, in the Appendix, we provide a "walk through" of the computations for two of the 32 groups we consider.

Acknowledgments. The authors would like to thank Tom Farrell and Ian Leary for many helpful comments on this project. The graphics in this paper were kindly produced by Dennis Burke. The authors are particularly grateful to Bruce Magurn for his extensive help with the computations of the algebraic $K$-theory of finite groups appearing in Section 5 of this paper, and for catching some errors in a preliminary version of this work. This project was partially supported by the NSF, under the grants DMS-0606002 and DMS-0805605. The first author was partly supported by an Alfred P. Sloan research fellowship.

\section{The 3-dimensional groups}

A hyperbolic Coxeter $n$-simplex $\Delta^{n}$ is an $n$-dimensional geodesic simplex in $\mathbb{H}^{n}$, all of whose dihedral angles are integral submultiples of $\pi$. We allow a simplex in $\mathbb{H}^{n}$ to be unbounded with ideal vertices on the sphere at infinity of $\mathbb{H}^{n}$. It is known that such simplices exist only in dimensions $n=2,3, \ldots, 9$, and that for $n \geq 3$, there are exactly 72 hyperbolic Coxeter simplices up to congruence (see for instance the discussion in [JKRT99] and [JKRT02]). 
A hyperbolic Coxeter $n$-simplex reflection group $\Gamma$ is the group generated by reflections in the sides of a Coxeter $n$-simplex in hyperbolic $n$-space $\mathbb{H}^{n}$. We will call such group a hyperbolic n-simplex group.

According to Vinberg [V67], the associated hyperbolic $n$-simplex groups of all but eight of the 72 simplices are arithmetic. The nonarithmetic groups are the hyperbolic Coxeter tetrahedra groups $[(3,4,3,5)][5,3,6] .\left[5,3^{[3]}\right],\left[\left(3^{3}, 6\right)\right],[(3,4,3,6)]$, $[(3,5,3,6)]$, and the 5-dimensional hyperbolic Coxeter group $\left[\left(3^{5}, 4\right)\right]$.

In dimension 3, there are 32 hyperbolic Coxeter tetrahedra groups; 9 of them are cocompact (see Figure 1), and 23 are noncocompact (see Figure 2). Let us briefly recall how the algebra and geometry of these groups are encoded in the Coxeter diagrams.

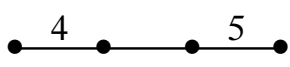

$[4,3,5]$

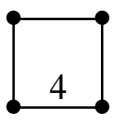

$\left[\left(3^{3}, 4\right)\right]$

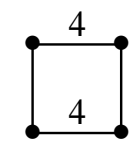

$\left[(3,4)^{[2]}\right]$

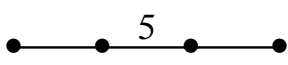

$[3,5,3]$

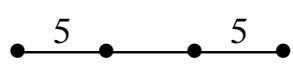

$[5,3,5]$

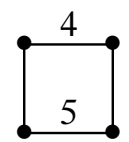

$[(3,4,3,5)]$

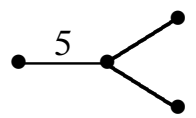

$\left[5,3^{1,1}\right]$

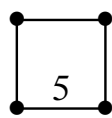

$\left[\left(3^{3}, 5\right)\right]$

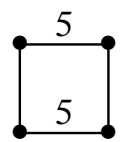

$\left[(3,5)^{[2]}\right]$

Figure 1. Cocompact hyperbolic Coxeter tetrahedral groups.

From the algebraic viewpoint, the Coxeter diagram encodes a presentation of the associated group $\Gamma$ as follows: associate a generator $x_{i}$ to each vertex $v_{i}$ of the Coxeter diagram (hence all of our groups will come equipped with four generators, as the Coxeter diagrams have four vertices). For the relations in $\Gamma$, one has the following:

(1) for every vertex $v_{i}$, one inserts the relation $x_{i}^{2}=1$;

(2) if two vertices $v_{i}, v_{j}$ are not joined by an edge, one inserts the relation $\left(x_{i} x_{j}\right)^{2}=$ 1 (so combined with the previous relation, one sees that $x_{i}$ and $x_{j}$ commute, generating a $\mathbb{Z} / 2 \times \mathbb{Z} / 2$ );

(3) if two vertices $v_{i}, v_{j}$ are joined by an unlabelled edge, one inserts the relation $\left(x_{i} x_{j}\right)^{3}=1$ (and in particular, the two elements $x_{i}, x_{j}$ generate a subgroup isomorphic to the dihedral group $D_{3}$ ); 


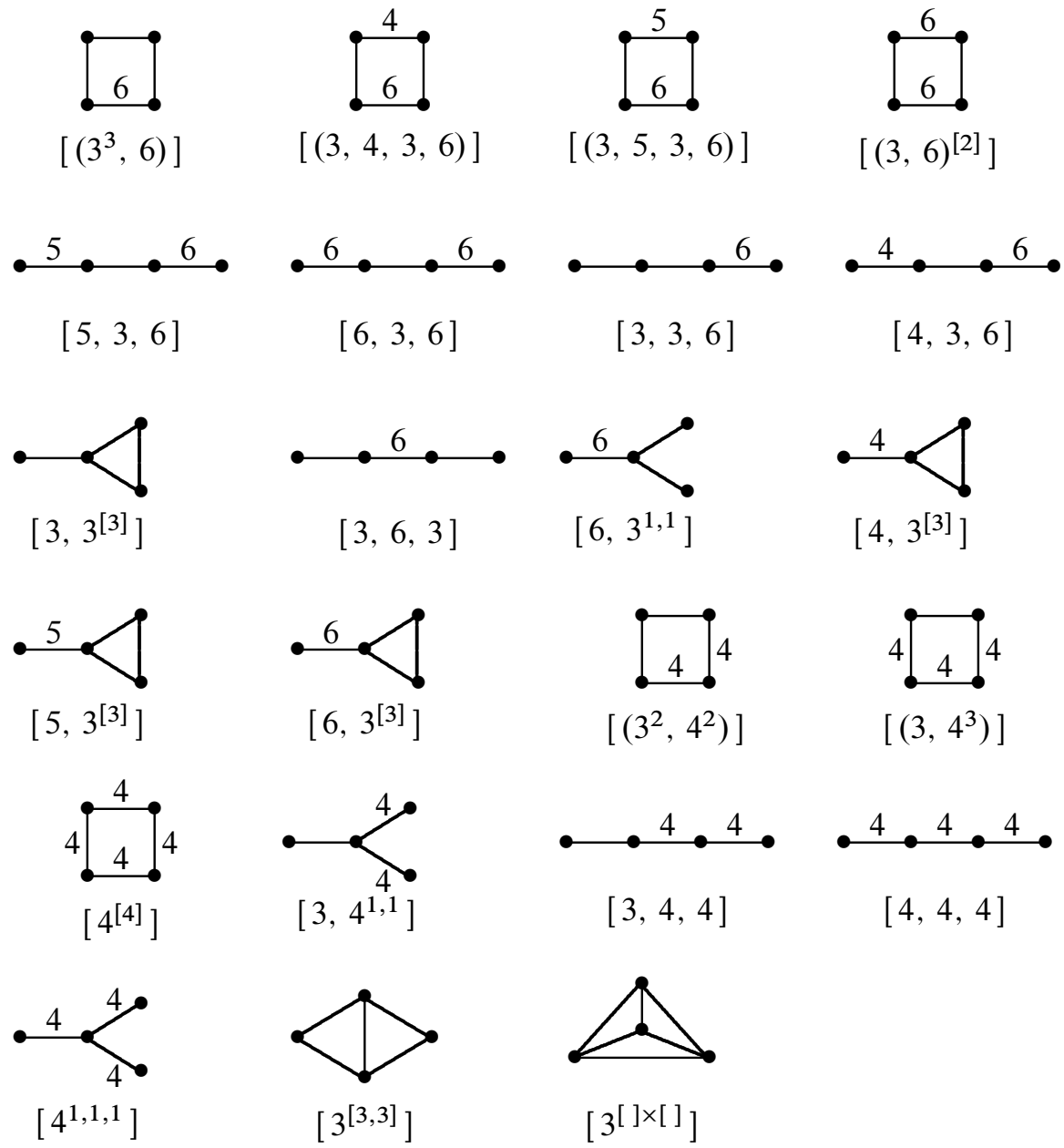

Figure 2. Noncocompact hyperbolic Coxeter tetrahedral groups.

(4) if two vertices $v_{i}, v_{j}$ are joined by an edge with label $m_{i j}$, one inserts the relation $\left(x_{i} x_{j}\right)^{m_{i j}}=1$ (and hence, the two elements $x_{i}, x_{j}$ generate a subgroup isomorphic to the dihedral group $D_{m_{i j}}$ ).

A special subgroup of $\Gamma$ will be a subgroup generated by a subset of the generating set. Observe that such a subgroup will automatically be a Coxeter group, with a presentation that can again be read off from the Coxeter diagram. Special subgroups generated by a pair of generators will always be isomorphic to a (finite) dihedral group. An important point for our purposes is that in our Coxeter groups, every finite subgroup can be conjugated into a finite special subgroup. In particular, since 
there are only finitely many special subgroups, one can quite easily classify up to isomorphism all the finite subgroups appearing in any of our 32 Coxeter groups.

Now let us move to the geometric viewpoint. As we mentioned earlier, associated to any of our 32 Coxeter groups, one has a simplex $\Delta^{3}$ in hyperbolic 3-space $\mathbb{H}^{3}$. Each of the four generators $x_{i}$ of the Coxeter group $\Gamma$ is bijectively associated with the hyperplane $P_{i}$ extending one of the four faces of the simplex $\Delta^{3}$, and the (interior) angles between the respective hyperplanes can again be read off from the Coxeter diagram:

(1) if two vertices $v_{i}, v_{j}$ are not joined by an edge, then $\angle\left(P_{i}, P_{j}\right)=\pi / 2$,

(2) if two vertices $v_{i}, v_{j}$ are joined by an unlabelled edge, then $\angle\left(P_{i}, P_{j}\right)=\pi / 3$,

(3) if two vertices $v_{i}, v_{j}$ are joined by an edge with label $m_{i j}$, then $\angle\left(P_{i}, P_{j}\right)=$ $\pi / m_{i j}$.

The resulting configuration of four hyperplanes exists, and is unique up to isometries of $\mathbb{H}^{3}$. One can now define the map $\Gamma \rightarrow O^{+}(3,1)=\operatorname{Isom}\left(\mathbb{H}^{3}\right)$ by sending each generator $x_{i}$ to the isometry obtained by reflecting in the corresponding hyperplane $P_{i}$. The condition on the angles between the hyperplanes ensures that this map respects the relations in $\Gamma$, and hence is actually a homomorphism. In fact this map is an embedding of $\Gamma$ as a discrete subgroup of $O^{+}(3,1)$, with fundamental domain for the associated action on $\mathbb{H}^{3}$ consisting precisely of the simplex $\Delta^{3}$.

Finally, to relate the geometric with the algebraic viewpoint, we remind the reader of the following bijective identifications:

(1) Given an edge in the 3 -simplex $\Delta^{3}$, lying on the intersection of two hyperplanes $P_{i}, P_{j}$, the subgroup of $\Gamma$ that fixes the edge pointwise is precisely the special subgroup $\left\langle x_{i}, x_{j}\right\rangle$ (and hence will be a dihedral group).

(2) Given a vertex in the 3-simplex $\Delta^{3}$, obtained as the intersection of three hyperplanes $P_{i}, P_{j}, P_{k}$, the subgroup of $\Gamma$ that stabilizes the vertex is precisely the special subgroup $\left\langle x_{i}, x_{j}, x_{k}\right\rangle$.

We point out that the stabilizer of a vertex of $\Delta^{3}$ will either be a finite Coxeter group (if the vertex lies inside $\mathbb{H}^{3}$ ), or will be a 2-dimensional crystallographic group (if the vertex is an ideal vertex). Furthermore, one can readily determine whether a vertex will be ideal or not, just by determining whether the associated special subgroup is crystallographic or finite.

It is known that for all the groups listed above the Farrell and Jones Isomorphism Conjecture in lower algebraic $K$-theory holds, that is $H_{n}^{\Gamma}\left(E_{\mathcal{V C}}(\Gamma) ; \mathbb{K} \mathbb{Z}^{-\infty}\right) \cong$ $K_{n}(\mathbb{Z} \Gamma)$ for $n<2$. This follows immediately from results in [FJ93] and [BFPP00], see the discussion in [Or04, pg. 325]. Our plan is to use this result to explicitly compute the lower algebraic $K$-theory of the integral group ring $\mathbb{Z} \Gamma$, for all of the 32 groups listed above. 


\section{A formula for the algebraic $K$-theory}

In this section, we combine some recent work of Lück and Weiermann [LW] with some previous work of the authors [LO07] to establish the following:

Proposition 3.1. Let $\mathcal{F} \subset \widetilde{\mathcal{F}}$ be a nested pair of families of subgroups of $\Gamma$, and assume that the collection of subgroups $\left\{H_{\alpha}\right\}_{\alpha \in I}$ is adapted to the pair $(\widetilde{F}, \widetilde{\mathcal{F}})$. Let $\mathscr{H}$ be a complete set of representatives of the conjugacy classes within $\left\{H_{\alpha}\right\}$, and consider the cellular $\Gamma$-pushouts:

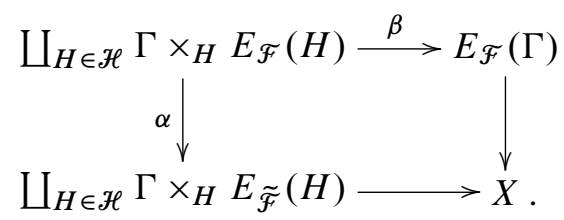

Then $X$ is a model for $E_{\widetilde{F}}(\Gamma)$. In the above cellular $\Gamma$-pushout, we require either (1) $\alpha$ is the disjoint union of cellular $H$-maps $(H \in \mathscr{H}$ ), $\beta$ is an inclusion of $\Gamma-C W$ complexes, or (2) $\alpha$ is the disjoint union of inclusions of $H$-CW-complexes $(H \in \mathscr{H})$, $\beta$ is a cellular $\Gamma$-map.

Proof. Let us start by recalling that a collection $\left\{H_{\alpha}\right\}_{\alpha \in I}$ of subgroups of $\Gamma$ is adapted to the pair $(\widetilde{F}, \widetilde{\mathcal{F}})$, provided that the following holds:

(1) For all $H_{1}, H_{2} \in\left\{H_{\alpha}\right\}_{\alpha \in I}$, either $H_{1}=H_{2}$, or $H_{1} \cap H_{2} \in \mathcal{F}$.

(2) The collection $\left\{H_{\alpha}\right\}_{\alpha \in I}$ is conjugacy closed i.e., if $H \in\left\{H_{\alpha}\right\}_{\alpha \in I}$ then $g H g^{-1} \in$ $\left\{H_{\alpha}\right\}_{\alpha \in I}$ for all $g \in \Gamma$.

(3) Every $H \in\left\{H_{\alpha}\right\}_{\alpha \in I}$ is self-normalizing, i.e., $N_{\Gamma}(H)=H$.

(4) For all $G \in \tilde{\mathcal{F}} \backslash \mathcal{F}$, there exists $H \in\left\{H_{\alpha}\right\}_{\alpha \in I}$ such that $G \leq H$.

Note that the subgroups in the collection $\left\{H_{\alpha}\right\}_{\alpha \in I}$ are not assumed to lie within the family $\widetilde{\mathcal{F}}$.

Using the existence of the adapted family $\left\{H_{\alpha}\right\}_{\alpha \in I}$, one can now define an equivalence relation on the subgroups in $\widetilde{\mathcal{F}}-\widetilde{F}$ as follows: we decree that $G_{1} \sim G_{2}$ if there exists an $H \in\left\{H_{\alpha}\right\}_{\alpha \in I}$ such that $G_{1} \leq H$ and $G_{2} \leq H$. Note that $\sim$ is indeed an equivalence relation: the symmetric property is immediate, while reflexivity follows from property (4) of adapted collection, and transitivity comes from property (1) of adapted collection. Furthermore this equivalence relation has the following two properties:

- if $G_{1}, G_{2} \in \widetilde{\mathcal{F}}-\mathcal{F}$ satisfies $G_{1} \leq G_{2}$, then $G_{1} \sim G_{2}$ (immediate from the definition of $\sim$ ).

- if $G_{1}, G_{2} \in \tilde{\mathcal{F}}-\mathcal{F}$ and $g \in \Gamma$, then $G_{1} \sim G_{2} \Leftrightarrow g G_{1} g^{-1} \sim g G_{2} g^{-1}$ (follows from property (2) of adapted collection). 
We denote by $[\tilde{\mathcal{F}}-\mathcal{F}]$ the set of equivalence classes of elements in $\widetilde{\mathcal{F}}-\mathscr{F}$ under the above equivalence relation, and for $G \in \widetilde{\mathcal{F}}-\mathscr{F}$, we will write $[G]$ for the corresponding equivalence class. Note that by the second property above, the $\Gamma$-action by conjugation on $\widetilde{\mathcal{F}}-\widetilde{F}$ preserves equivalence classes, and hence descends to a $\Gamma$ action on $[\widetilde{\mathcal{F}}-\widetilde{F}]$. We let $I$ be a complete set of representatives $[G]$ of the $\Gamma$-orbits in $[\widetilde{\mathscr{F}}-\widetilde{F}]$. For $G \in \widetilde{\mathcal{F}}-\widetilde{F}$, define the subgroup

$$
N_{\Gamma}[G]:=\left\{g \in \Gamma \mid\left[g G g^{-1}\right]=[G]\right\},
$$

which is precisely the isotropy group of $[G] \in[\widetilde{\mathcal{F}}-\mathscr{F}]$ under the $\Gamma$-action induced by conjugation. Finally, define a family of subgroups $\widetilde{\mathcal{F}}[G]$ of the group $N_{\Gamma}[G]$ by

$$
\widetilde{\mathcal{F}}[G]:=\left\{K \subset N_{\Gamma}[G] \mid K \in \widetilde{\mathscr{F}}-\mathscr{F},[K]=[G]\right\} \cup\left\{K \subset N_{\Gamma}[G] \mid K \in \mathscr{F}\right\} .
$$

Observe that the notions defined above (introduced in [LW]) make sense for any equivalence relation on $\widetilde{\mathcal{F}}-\widetilde{F}$ satisfying the two properties mentioned above.

Now [LW, Theorem 2.3] states that for any equivalence relation $\sim$ on the elements in $\widetilde{\mathcal{F}}-\widetilde{F}$ satisfying the two properties above (and with the notation used in the previous paragraph), the $\Gamma$-CW-complex $X$ defined by the cellular $\Gamma$-pushout depicted below is a model for $E_{\widetilde{F}}(\Gamma)$.

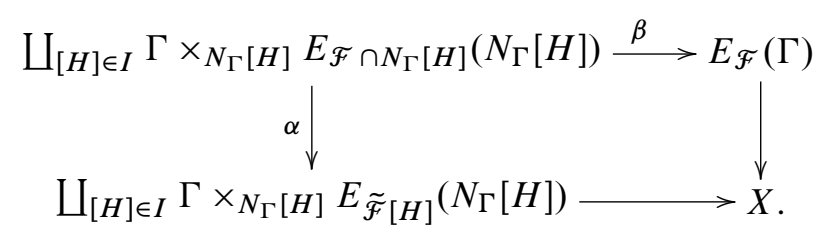

In the above cellular $\Gamma$-pushout, Lück-Weiermann require either (1) $\alpha$ is the disjoint union of cellular $N_{\Gamma}[H]$-maps $([H] \in I), \beta$ is an inclusion of $\Gamma$-CW-complexes, or (2) $\alpha$ is the disjoint union of inclusions of $N_{\Gamma}[H]$-CW-complexes $([H] \in I), \beta$ is a cellular $\Gamma$-map.

We now proceed to verify that, for the equivalence relation we have defined using the adapted family $\left\{H_{\alpha}\right\}_{\alpha \in I}$, the left hand terms in the cellular $\Gamma$-pushout given above reduce to precisely the left hand terms appearing in the statement of our proposition. This boils down to two claims:

Claim 1: For any $G \in \widetilde{\mathcal{F}}-\mathcal{F}$, we have the equality $N_{\Gamma}[G]=H$ where $H$ is the unique element in $\left\{H_{\alpha}\right\}_{\alpha \in I}$ satisfying $G \leq H$.

To see this, we first note that there indeed is a unique $H \in\left\{H_{\alpha}\right\}_{\alpha \in I}$ satisfying $G \leq H$, for if there were two such groups $H_{1} \neq H_{2}$, then we would immediately see that $H_{1} \cap H_{2} \geq G \in \widetilde{\mathcal{F}}-\mathcal{F}$, contradicting the property (1) of an adapted collection. Next we observe that if $h \in H$, then $h G h^{-1} \leq h H h^{-1}=H$, and hence 
that $\left[h G h^{-1}\right]=[G]$, which implies the containment $H \leq N_{\Gamma}[G]$. Conversely, if $k \in N_{\Gamma}[G]$, then we have that $[G]=\left[k G k^{-1}\right]$, and so from the definition of the equivalence relation there must exist some $\bar{H} \in\left\{H_{\alpha}\right\}_{\alpha \in I}$ with $G \leq \bar{H}$ and $k G k^{-1} \leq \bar{H}$. Since we already know that $G \leq H$, the uniqueness forces $\bar{H}=H$, and thus that $k G k^{-1} \leq H$. This in turn tells us that $H \cap k^{-1} H k \geq G \in \widetilde{\mathcal{F}}-\mathscr{F}$, and property (1) of an adapted collection now forces $H=k^{-1} H k$, which implies that $k \in N_{\Gamma}(H)$. But property (3) of an adapted collection forces the group $H$ to be self-normalizing, giving $k \in H$, and completing the proof of the reverse inclusion.

Claim 2: For any $G \in \underset{\mathscr{F}}{\widetilde{F}}-\widetilde{F}$, the family $\underset{\mathcal{F}}{\widetilde{\mathscr{F}}}[G]$ on the group $N_{\Gamma}[G]=H$ (see the previous claim) coincides with the restriction $\widetilde{\mathscr{F}} \cap H$ of the family $\widetilde{\mathscr{F}}$ to the subgroup $H$ (i.e., consisting of all elements in $\widetilde{\mathcal{F}}$ that lie within $H$ ).

Note that the containment $\widetilde{\mathcal{F}}[G] \subset \widetilde{\mathscr{F}} \cap H$ is obvious from the definition of $\widetilde{\mathcal{F}}[G]$. For the opposite containment, let $K \in \widetilde{\mathscr{F}} \cap H \subset \widetilde{\mathscr{F}}$, and observe that $K \leq H$ and either $K \in \mathscr{F}$, or $K \in \widetilde{\mathcal{F}}-\mathcal{F}$. In the first case, we have $K \in \mathscr{F} \cap H \subset \widetilde{\mathcal{F}}[G]$, while in the second case, we have that $[K]=[G]$ by the definition of the equivalence relation, and hence again we have $K \in \widetilde{\widetilde{F}}[G]$. This gives us the containment $\widetilde{\mathcal{F}} \cap H \subset$ $\widetilde{\widetilde{F}}[G]$, giving us the claim.

Having established our two claims, we can now substitute the expressions from the claims for the corresponding ones in the Lück-Weiermann diagram. Finally, we comment on the indices in the disjoint sums appearing in the right hand of the diagrams. In the expression of Lück-Weiermann, the disjoint sum is taken over $I$, a complete system of representatives $[G]$ of the $\Gamma$-orbits in $[\widetilde{\mathcal{F}}-\mathcal{F}]$. But observe that from the definition of the equivalence relation we are using, classes in $[\widetilde{\mathscr{F}}-\widetilde{F}]$ can be bijectively identified with groups $H \in\left\{H_{\alpha}\right\}_{\alpha \in I}$ (by associating each class in $[\widetilde{\mathcal{F}}-\widetilde{F}]$ with the unique element in $\left\{H_{\alpha}\right\}_{\alpha \in I}$ containing all the elements in the class). Since it is clear that the $\Gamma$-action on $[\widetilde{\mathcal{F}}-\mathcal{F}]$ coincides (under the bijection above) with the $\Gamma$-action on the set $\left\{H_{\alpha}\right\}_{\alpha \in I}$, we can replace the system of representatives $I$ by the system of representatives $\mathscr{H}$. This completes the proof of the proposition.

We now specialize to the case where $\mathscr{F}=\mathcal{F} \mathcal{I} \mathcal{N}$ and $\widetilde{\mathcal{F}}=\mathcal{V C}$, and obtain the following:

Corollary 3.2. Given the group $\Gamma$, assume that the collection of subgroups $\left\{H_{\alpha}\right\}_{\alpha \in I}$ is adapted to the pair $(\mathcal{F} \mathcal{I} \mathcal{N}, \mathcal{V} C)$. If $\mathscr{H}$ be a complete set of representatives of the conjugacy classes within $\left\{H_{\alpha}\right\}$, then we have a splitting

$$
\begin{aligned}
& H_{*}^{\Gamma}\left(E_{\mathcal{V C}}(\Gamma) ; \mathbb{K}^{-\infty}\right) \\
& \quad \cong H_{*}^{\Gamma}\left(E_{\mathcal{F} \mathcal{I N}}(\Gamma) ; \mathbb{K} \mathbb{Z}^{-\infty}\right) \oplus \bigoplus_{H \in \mathscr{H}} H_{*}^{H}\left(E_{\mathcal{F} \mathcal{I N}}(H) \rightarrow E_{\mathcal{V C}}(H)\right) .
\end{aligned}
$$


Proof. Let us work with the explicit model $X$ for $E_{\mathcal{V} \mathcal{C}}(\Gamma)$ constructed via the previous proposition. Since $X$ is obtained as a double mapping cylinder, there exists an obvious map $\rho: X \rightarrow[0,1]$, which further has the property that every point pre-image is $\Gamma$ invariant. In particular, corresponding to the splitting of $[0,1]$ into $[0,2 / 3) \cup(1 / 3,1]$, we get a $\Gamma$-invariant splitting of $X$. If we let $A=\rho^{-1}[0,2 / 3), B=\rho^{-1}(1 / 3,1]$, then from the Mayer-Vietoris sequence in equivariant homology (and omitting coefficients to simplify notation), we have that

$$
\cdots \longrightarrow H_{n}^{\Gamma}(A \cap B) \longrightarrow H_{n}^{\Gamma}(A) \oplus H_{n}^{\Gamma}(B) \longrightarrow H_{n}^{\Gamma}(X) \longrightarrow \cdots
$$

But now observe that we have obvious $\Gamma$-equivariant homotopy equivalences:

- $A=\rho^{-1}[0,2 / 3) \simeq \rho^{-1}(0)=\bigsqcup_{H \in \mathscr{H}} \Gamma \times_{H} E_{\mathcal{V C}}(H)$,

- $B=\rho^{-1}(1 / 3,1] \simeq \rho^{-1}(1)=E_{\mathscr{F} I \mathcal{N}}(\Gamma)$,

- $A \cap B=\rho^{-1}(1 / 3,2 / 3) \simeq \rho^{-1}(1 / 2)=\bigsqcup_{H \in \mathscr{H}} \Gamma \times_{H} E_{\mathcal{F} \mathcal{I N}}(H)$.

Now combining the fact that our equivariant generalized homology theory turns disjoint unions into direct sums, along with the induction structure, this allows us to evaluate the terms in the Mayer-Vietoris sequence

$$
\begin{aligned}
\cdots \longrightarrow & \bigoplus_{H \in \mathscr{H}} H_{n}^{H}\left(E_{\mathscr{F} \mathcal{I N}} H\right) \longrightarrow H_{n}^{\Gamma}\left(E_{\mathscr{F} \mathcal{L N}} \Gamma\right) \oplus \bigoplus_{H \in \mathscr{H}} H_{n}^{H}\left(E_{\mathcal{V} C} H\right) \\
& \longrightarrow H_{n}^{\Gamma}\left(E_{\mathcal{V} C} \Gamma\right) \longrightarrow \cdots .
\end{aligned}
$$

But recall that Bartels [Bar03] has established that for any group $G$, the relative assembly map

$$
H_{*}^{G}\left(E_{\mathscr{F} \mathcal{I N}}(G) ; \mathbb{K} \mathbb{Z}^{-\infty}\right) \rightarrow H_{*}^{G}\left(E_{\mathcal{V C}}(G) ; \mathbb{K} \mathbb{Z}^{-\infty}\right)
$$

is split injective. In particular, for each integer $n$, the above portion of the MayerVietoris long exact sequence breaks off as a short exact sequence (since the initial term injects). Since the map from the $H_{n}^{\Gamma}\left(E_{\mathcal{F} \mathcal{I N}} \Gamma\right) \rightarrow H_{n}^{\Gamma}\left(E_{\mathcal{V C}} \Gamma\right)$ is also split injective (from Bartels result), we immediately obtain an identification of the cokernel of this map with the cokernel of the map

$$
\bigoplus_{H \in \mathscr{H}} H_{n}^{H}\left(E_{\mathcal{F} \mathcal{I N}} H\right) \rightarrow \bigoplus_{H \in \mathscr{H}} H_{n}^{H}\left(E_{\mathcal{V C}} H\right)
$$

But from the definition of the map $\alpha$ in Proposition 3.1, we see that the latter map splits as a direct sum (over $H \in \mathscr{H}$ ) of the relative assembly maps $H_{n}^{H}\left(E_{\mathcal{F} \mathcal{I N}} H\right) \rightarrow$ $H_{n}^{H}\left(E_{\mathcal{V C}} H\right)$. This immediately yields a corresponding splitting of the cokernel, completing the proof of the corollary.

Next we recall that the authors established in [LO07, Theorem 2.6] that in the case where $\Gamma$ is hyperbolic relative to a collection of subgroups $\left\{H_{i}\right\}_{i=1}^{k}$ (assumed to be pairwise non-conjugate), then the collection of subgroups consisting of 
(1) all conjugates of $H_{i}$ (these will be called peripheral subgroups),

(2) all maximal infinite virtually cyclic subgroups $V$ such that $V \nsubseteq g H_{i} g^{-1}$, for all $i=1, \cdots k$, and for all $g \in \Gamma$,

is adapted to the pair of families $(\mathcal{F} \mathcal{I} \mathcal{N}, \mathcal{V C})$. Applying the previous corollary to this special case, we get:

Corollary 3.3. Assume that the group $\Gamma$ is hyperbolic relative to the collection of subgroups $\left\{H_{i}\right\}_{i=1}^{k}$ (assumed to be pairwise nonconjugate). Let $\mathcal{V}$ be a complete set of representatives of the conjugacy classes of maximal infinite virtually cyclic subgroups inside $\Gamma$ which cannot be conjugated within any of the $H_{i}$. Then we have a splitting

$$
\begin{aligned}
H_{*}^{\Gamma}\left(E_{\mathcal{V C}}(\Gamma) ; \mathbb{K}^{-\infty}\right) \cong & H_{*}^{\Gamma}\left(E_{\mathcal{F} \mathcal{I N}}(\Gamma) ; \mathbb{K} \mathbb{Z}^{-\infty}\right) \\
& \oplus \bigoplus_{i=1}^{k} H_{*}^{H_{i}}\left(E_{\mathcal{F} \mathcal{I N}}\left(H_{i}\right) \rightarrow E_{\mathcal{V C}}\left(H_{i}\right)\right) \\
& \oplus \bigoplus_{V \in \mathcal{V}} H_{*}^{V}\left(E_{\mathcal{F} \mathcal{I N}}(V) \rightarrow *\right) .
\end{aligned}
$$

The primary example of relatively hyperbolic groups are groups $\Gamma$ acting with cofinite volume (but not cocompactly) on a complete, simply connected, Riemannian manifold whose sectional curvature satisfies $-b^{2} \leq K \leq-a^{2}<0$. These groups are hyperbolic relative to the "cusp groups", which one can take to be the infinite subgroups arising as stabilizers of ideal points in the boundary at infinity of the Riemannian manifold. We note that non-uniform lattices in $O^{+}(n, 1)=\operatorname{Isom}\left(\mathbb{H}^{n}\right)$ are examples of relatively hyperbolic groups, and for this class of groups, the cusp groups are automatically $(n-1)$-dimensional crystallographic groups (this is due to the fact that the horospheres have intrinsic geometry homothetic to $\mathbb{R}^{n-1}$ ). Observe that 23 of the groups we are considering (see Figure 2) are non-uniform lattices in $O^{+}(3,1)$, and hence are relatively hyperbolic groups, relative to a collection of subgroups, each of which is isomorphic to a 2-dimensional crystallographic group. For these 23 groups, the situation is even further simplified by the following observations:

- Pearson [Pe98] showed that for any 2-dimensional crystallographic group $H$, the relative assembly map is an isomorphism for $n \leq 1$, and hence that

$$
H_{n}^{H}\left(E_{\mathcal{F} \mathcal{L N}}(H) \rightarrow E_{\mathcal{V C}}(H)\right)=0
$$

for $n \leq 1$.

- The authors in [LO07, Section 3] gave a general procedure for classifying the maximal virtually cyclic subgroups of Coxeter groups acting on $\mathbb{H}^{3}$. The groups fall into three types, with infinitely many conjugacy classes of type II and type III, 
and only finitely many conjugacy classes of type I subgroups. Furthermore, the relative assembly map is an isomorphism (for $n \leq 1$ ) for all groups of type II and III. This is discussed in more detail in Section 4.

- Work of Farrell and Jones [FJ93] and Berkove, Farrell, Juan-Pineda, and Pearson [BFPP00] implies that the Farrell-Jones isomorphism conjecture holds for all lattices $\Gamma$ in hyperbolic space (and $k \leq 1$ ), and hence that one has isomorphisms

$$
K_{n}(\mathbb{Z} \Gamma) \cong H_{n}^{\Gamma}\left(E_{\mathcal{V C}}(\Gamma) ; \mathbb{K} \mathbb{Z}^{-\infty}\right)
$$

for all $n \leq 1$.

Combining these observations with the previous corollary yields the following:

Corollary 3.4. Let $\Gamma \leq O^{+}(3,1)$ be any Coxeter group arising as a lattice (uniform or non-uniform), and let $\left\{V_{i}\right\}_{i=1}^{k}$ be a complete set of representatives for conjugacy classes of type I maximal virtually infinite cyclic subgroups of $\Gamma$. Then we have, for all $n \leq 1$, isomorphisms

$$
K_{n}(\mathbb{Z} \Gamma) \cong H_{n}^{\Gamma}\left(E_{\mathcal{F} \mathcal{I N}}(\Gamma) ; \mathbb{K} \mathbb{Z}^{-\infty}\right) \oplus \bigoplus_{i=1}^{k} H_{n}^{V_{i}}\left(E_{\mathcal{F} \mathcal{I N}}\left(V_{i}\right) \rightarrow *\right)
$$

In the next sections, we will implement this corollary to compute the lower algebraic $K$-theory of the integral group rings of all 32 of the 3-simplex hyperbolic reflection groups.

\section{Maximal infinite $v \mathcal{C}_{\infty}$ subgroups}

In this section, we proceed to classify the maximal infinite virtually cyclic $\left(\mathcal{V} C_{\infty}\right)$ subgroups arising in our groups. Let us start by briefly recalling some of the results from Section 3 of [LO07]. First of all, for a lattice $\Gamma$ in $O^{+}(n, 1)$, infinite $\mathcal{V C}$ subgroups are of two types: those that fix a single point in the boundary at infinity, and those that fix a pair of points in the boundary at infinity. We call subgroups of the first type parabolic, and those of second type hyperbolic. Note that every parabolic subgroup can be conjugated into a cusp group; for the purpose of our classification, we will ignore these subgroups. The subgroups of hyperbolic type automatically stabilize the geodesic joining the pair of fixed points in the boundary at infinity. Furthermore the geodesic they stabilize will project to a periodic curve in the quotient space $\mathbb{H}^{n} / \Gamma$. Note that conversely, stabilizers of periodic geodesics are infinite $\mathcal{V} \mathcal{C}$ subgroups of $\Gamma$. This implies that the maximal hyperbolic type infinite $\mathcal{V} C$ subgroups of $\Gamma$ are in bijective correspondence with stabilizers of periodic geodesics.

We now specialize to the case where $n=3$, and $\Gamma$ is a Coxeter group. In this situation, we can subdivide the family of periodic geodesics into three types. 
- A geodesic whose projection has non-trivial intersection with the interior of the polyhedron $\mathbb{H}^{3} / \Gamma$, which we call type III.

- A geodesic whose projection lies in the boundary of the polyhedron $\mathbb{H}^{3} / \Gamma$, but does not lie inside the 1-skeleton of $\mathbb{H}^{3} / \Gamma$, which we call type II.

- A geodesic whose projection lies in the 1-skeleton of the polyhedron $\mathbb{H}^{3} / \Gamma$, which we call type I.

For geodesics of type III, it is easy to see that the stabilizer of the geodesic must be isomorphic to either $\mathbb{Z}$ or $D_{\infty}$. For geodesics of type II, the stabilizer is always isomorphic to either $\mathbb{Z}_{2} \times \mathbb{Z}$ or $\mathbb{Z}_{2} \times D_{\infty}$. The main purpose of this section will be to classify stabilizers of type I geodesics for all 32 groups which occur as hyperbolic 3 -simplex reflection groups.

We start by outlining our approach: all the groups we are considering have fundamental domain consisting of a 3-dimensional simplex in $\mathbb{H}^{3}$ (possibly with some ideal vertices). So up to conjugacy, for each of the groups we are considering, we can have at most six distinct stabilizers of type I geodesic (one for each edge in the fundamental domain, fewer in the presence of ideal vertices). But this is actually an overcount, as one could potentially have a type I geodesic whose projection into the fundamental domain passes through several of the edges. So the first step is to understand how many distinct stabilizers (up to conjugacy) one obtains.

Let us explain how one can find out the number of distinct stabilizers. Note that, at every (non-ideal) vertex $v$ of our fundamental domain 3 -simplex in $\mathbb{H}^{3}$, we can consider a small $\varepsilon$-sphere $S_{v}$ centered at $v$. Now the tessellation of $\mathbb{H}^{3}$ by copies of the fundamental domain induces a tessellation of $S_{v}$ by isometric spherical triangles. In fact, the tessellation of $S_{v}$ is the one naturally associated with the special subgroup of the Coxeter group $\Gamma$ that stabilizes the vertex $v$. Now note that, if we were to label the three edges of the 3-simplex incident to $v$, we get a corresponding label of the three vertices of a spherical triangle in the tessellation of $S_{v}$. One can extend this labeling via reflections, both for the tessellation of $\mathbb{H}^{3}$ and the tessellation of $S_{v}$.

Now given a periodic geodesic of type I, with a portion of the geodesic projecting to the edge $e$ in the 3 -simplex, with $e$ adjacent to the vertex $v$, one can easily "read off" from the labeled tessellation of $S_{v}$ which edge extends the geodesic. Indeed, this will be picked up by the label of the vertex in the tessellation of $S_{v}$ which is antipodal to the labeled vertex corresponding to $e$. In this manner, one can easily decide the number of distinct stabilizers of type I geodesics that arise for the 32 groups we are considering.

To recognize the tessellations arising for the various $S_{v}$, one now notes that the isometry group of each of these tessellations can be obtained by looking at the stabilizer $\Gamma_{v}$ of the vertex $v$ in the group $\Gamma$. These stabilizers are finite special subgroups of the Coxeter group $\Gamma$, generated by three of the four canonical generators of $\Gamma$. From the classification of the 32 hyperbolic 3 -simplex groups, it is easy to list out all 
such finite special subgroups: there are eight of these, namely $\mathbb{Z}_{2} \times D_{2}, \mathbb{Z}_{2} \times D_{3}$, $\mathbb{Z}_{2} \times D_{4}, \mathbb{Z}_{2} \times D_{5}, \mathbb{Z}_{2} \times D_{6},[3,3] \cong S_{4},[3,4] \cong \mathbb{Z}_{2} \times S_{4}$, and [3,5] $\cong \mathbb{Z}_{2} \times A_{5}$.

The next step is to identify the stabilizers of the corresponding geodesics. In the situation we are considering, all the type I geodesics $\eta$ that appear have stabilizer $\operatorname{Stab}_{\Gamma}(\eta)$ acting with fundamental domain an interval. In fact, the interval can be identified with the quotient space $\eta / \operatorname{Stab}_{\Gamma}(\eta) \subset \mathbb{H}^{3} / \Gamma$, which will be a union of edges in the 1-skeleton of the 3-simplex $\mathbb{H}^{3} / \Gamma$. Hence the group $\operatorname{Stab}_{\Gamma}(\eta)$ can be identified using Bass-Serre theory: it will be the fundamental group of a graph of groups, where the graph of groups consists of a single edge joining two vertices, with edge/vertex groups which can be explicitly found from the tessellations. We will say that the geodesic (or sometimes the edge in the 1-skeleton) reflects at the two endpoint vertices.

Indeed, the edge group $G_{e}$ will be precisely the stabilizer of one of the edges in $\eta / \operatorname{Stab}_{\Gamma}(\eta) \subset \mathbb{H}^{3} / \Gamma$. On the other hand, the vertex groups $G_{v}, G_{w}$ can be found by looking at each of the two endpoint vertices $v, w$ for $\eta / \operatorname{Stab}_{\Gamma}(\eta)$, and studying the spherical tessellations of $S_{v}, S_{w}$. Note that we are trying to identify elements in $\Gamma$, which stabilize the vertex $v$ (respectively $w$ ), and additionally map the geodesic $\eta$ through $v$ to itself. In particular, it must map the pair of antipodal vertices $\eta^{ \pm}$ (corresponding to the incoming/outgoing $\eta$-directions) in the tessellation of $S_{v}$ to themselves. The subgroup $G_{e} \subset G_{v}$ can be identified with the index 2 subgroup consisting of elements $G_{v}$ which fix both of the points $\eta^{ \pm}$. Now there is an obvious map which permutes the two points $\eta^{+}$and $\eta^{-}$, namely the reflection in the equator equidistant from these two points. But it is not clear that this reflection preserves the tessellation of $S_{v}$; in some cases, one will need to reflect in the equator, and then rotate by a certain angle along the $\eta^{ \pm}$axis, in order to obtain an element in $\Gamma_{v}$. Note that if the reflection in the equator preserves the tessellation, then we immediately obtain that $G_{v} \cong G_{e} \times \mathbb{Z}_{2}$. If the reflection in the equator does not preserve the tessellation, then we obtain that $G_{v} \cong G_{e} \rtimes \mathbb{Z}_{2}$. One can perform the same analysis at the vertex $w$, and hence find an expression for $\operatorname{Stab}_{\Gamma}(\eta)$ as an amalgamation of the groups $G_{v}, G_{w}$ over the index 2 subgroups $G_{e}$.

We make two observations: first of all, the stabilizer of an edge will always be a special subgroup of $\Gamma$, generated by a pair of canonical generators in $\Gamma$. In particular, the group $G_{e}$ will always be a dihedral group $D_{k}$ for some $k$. Now the vertex groups are of two types: (1) if the reflection in the equator preserves the tessellation, we obtain $G_{v} \cong \mathbb{Z}_{2} \times D_{k}$, or (2) if the reflection in the equator does not preserve the tessellation, then one can explicitly read off the semi-direct product structure from the tessellation, and in fact it is easy to see that $G_{v} \cong D_{2 k}$. In Table 1 below, we list out, for each of the finite special subgroups we need to consider, the edges that reflect, as well as the corresponding $G_{v}$. Let us explain the notation used in the table: the first column gives the various finite special subgroups that occur, the second column lists the angles that appear in the spherical triangles of the corresponding tessellation 
of $S_{v}$. The remaining three columns are ordered from smallest angle to largest, and expresses whether (1) the corresponding edge extends (i.e., does not reflect) at $v$, and (2) if it reflects, the corresponding subgroup $G_{v}$.

Table 1. Finite special subgroups \& local behavior of edges.

\begin{tabular}{|c|c|c|c|c|}
\hline $\mathbb{Z}_{2} \times D_{2}$ & $\pi / 2, \pi / 2, \pi / 2$ & $\mathbb{Z}_{2} \times D_{2}$ & $\mathbb{Z}_{2} \times D_{2}$ & $\mathbb{Z}_{2} \times D_{2}$ \\
\hline $\mathbb{Z}_{2} \times D_{3}$ & $\pi / 3, \pi / 2, \pi / 2$ & $\mathbb{Z}_{2} \times D_{3}$ & extends & extends \\
\hline $\mathbb{Z}_{2} \times D_{4}$ & $\pi / 4, \pi / 2, \pi / 2$ & $\mathbb{Z}_{2} \times D_{4}$ & $\mathbb{Z}_{2} \times D_{2}$ & $\mathbb{Z}_{2} \times D_{2}$ \\
\hline $\mathbb{Z}_{2} \times D_{5}$ & $\pi / 5, \pi / 2, \pi / 2$ & $\mathbb{Z}_{2} \times D_{5}$ & extends & extends \\
\hline $\mathbb{Z}_{2} \times D_{6}$ & $\pi / 6, \pi / 2, \pi / 2$ & $\mathbb{Z}_{2} \times D_{6}$ & $\mathbb{Z}_{2} \times D_{2}$ & $\mathbb{Z}_{2} \times D_{2}$ \\
\hline$S_{4}$ & $\pi / 3, \pi / 3, \pi / 2$ & extends & extends & $D_{4}$ \\
\hline $\mathbb{Z}_{2} \times S_{4}$ & $\pi / 4, \pi / 3, \pi / 2$ & $\mathbb{Z}_{2} \times D_{4}$ & $D_{6}$ & $\mathbb{Z}_{2} \times D_{2}$ \\
\hline $\mathbb{Z}_{2} \times A_{5}$ & $\pi / 5, \pi / 3, \pi / 2$ & $D_{10}$ & $D_{6}$ & $\mathbb{Z}_{2} \times D_{2}$ \\
\hline
\end{tabular}

From the Coxeter diagrams of the 32 groups we are considering, we can now read off quite easily the number (up to conjugacy) of stabilizers of type I geodesics. We now proceed to summarize the results of this procedure, which we list out in Tables 2,3 , and 4 . We remind the reader that, in addition to these subgroups, there will also be (up to conjugacy) countably infinitely many maximal $\mathcal{V} C$ subgroup of hyperbolic type isomorphic to one of $\mathbb{Z}, D_{\infty}, \mathbb{Z}_{2} \times \mathbb{Z}, \mathbb{Z}_{2} \times D_{\infty}$ (coming from stabilizers of type II and type III geodesics). The list below can be thought of as the "exceptional" maximal $\mathcal{V} C$ subgroups of hyperbolic type. Indeed, as we will see in the subsequent sections, these will be the only maximal $\mathcal{V} C$ subgroups of hyperbolic type that will actually contribute to the algebraic $K$-theory of the ambient groups.

4.1. The uniform lattices. There are 9 hyperbolic 3 -simplex groups with fundamental domain a compact 3 -simplex in $\mathbb{H}^{3}$. The number and type of stabilizers of type I geodesics are listed in the following table:

Table 2. Structure of $\mathcal{V} \mathcal{C}$ subgroups of cocompact groups.

\begin{tabular}{|c|c|}
\hline$\Gamma$ & $\operatorname{Stab}_{\Gamma}$ \\
\hline$\left[\left(3^{3}, 5\right)\right]$ & $D_{3} \times D_{\infty}, D_{5} \times D_{\infty},\left(D_{2} \times \mathbb{Z}_{2}\right) *_{D_{2}} D_{4}$ (twice) \\
\hline$[5,3,5]$ & $D_{2} \times D_{\infty}, D_{3} \times D_{\infty}, D_{5} \times D_{\infty}$ (twice) \\
\hline$\left[\left(3^{3}, 4\right)\right]$ & $D_{3} \times D_{\infty}, D_{4} \times D_{\infty},\left(D_{2} \times \mathbb{Z}_{2}\right) *_{D_{2}} D_{4}$ (twice) \\
\hline$[3,5,3]$ & $D_{2} \times D_{\infty}, D_{3} \times D_{\infty}$ (twice),$D_{5} \times D_{\infty}$ \\
\hline
\end{tabular}




\begin{tabular}{|c|c|}
\hline$\Gamma$ & $\mathrm{Stab}_{\Gamma}$ \\
\hline$\left[5,3^{1,1}\right]$ & $D_{2} \times D_{\infty}($ twice $), D_{3} \times D_{\infty}, D_{5} \times D_{\infty},\left(D_{2} \times \mathbb{Z}_{2}\right) * D_{2} D_{4}$ \\
\hline$[4,3,5]$ & $D_{2} \times D_{\infty}($ twice $), D_{3} \times D_{\infty}, D_{4} \times D_{\infty}, D_{5} \times D_{\infty}$ \\
\hline$\left[(3,5)^{[2]}\right]$ & $D_{2} \times D_{\infty}$ (twice) $, D_{3} \times D_{\infty}($ twice $), D_{5} \times D_{\infty}($ twice $)$ \\
\hline$[(3,4,3,5)]$ & $D_{2} \times D_{\infty}$ (twice),$D_{3} \times D_{\infty}$ (twice), $D_{4} \times D_{\infty}, D_{5} \times D_{\infty}$ \\
\hline$\left[(3,4)^{[2]}\right]$ & $D_{2} \times D_{\infty}$ (twice),$D_{3} \times D_{\infty}$ (twice), $D_{4} \times D_{\infty}$ (twice) \\
\hline
\end{tabular}

4.2. One ideal vertex. We have nine such Coxeter groups, namely the groups $\left[5,3^{[3]}\right],[5,3,6],\left[3^{2}, 4^{2}\right],\left[4,3^{[3]}\right],\left[3,3^{[3]}\right],\left[3,4^{1,1}\right],[4,3,6],[3,3,6]$, and $[3,4,4]$. The number and type of (non-finite) stabilizers of type I geodesics, as well as the cusp subgroups are listed in the following table:

Table 3. Structure of $\mathcal{V} \mathcal{C}$ subgroups of 1-ideal vertex groups.

\begin{tabular}{|c|c|c|c|}
\hline$\Gamma$ & & $\operatorname{Stab}_{\Gamma}$ & Cusp \\
\hline$\left[3,3^{[3]}\right]$ & 1 edge & $D_{4} * D_{2} D_{4}$ & {$\left[3^{[3]}\right]$} \\
\hline$[3,3,6]$ & 1 edge & $\left(D_{2} \times \mathbb{Z}_{2}\right) * D_{2} D_{4}$ & {$[3,6]$} \\
\hline$\left[5,3^{[3]}\right]$ & 2 edges & $D_{2} \times D_{\infty}, \quad D_{5} \times D_{\infty}$ & {$\left[3^{[3]}\right]$} \\
\hline$[5,3,6]$ & 2 edges & $D_{2} \times D_{\infty}, \quad D_{5} \times D_{\infty}$ & {$[3,6]$} \\
\hline$\left[\left(3^{2}, 4^{2}\right)\right]$ & 2 edges & $D_{2} \times D_{\infty}, \quad D_{3} \times D_{\infty}$ & {$[4,4]$} \\
\hline$\left[4,3^{[3]}\right]$ & 2 edges & $D_{2} \times D_{\infty}, \quad D_{4} \times D_{\infty}$ & {$\left[3^{[3]}\right]$} \\
\hline$[3,4,4]$ & 2 edges & $D_{2} \times D_{\infty}, \quad D_{3} \times D_{\infty}$ & {$[4,4]$} \\
\hline$\left[3,4^{1,1}\right]$ & 3 edges & $D_{2} \times D_{\infty}($ twice $), \quad D_{3} \times D_{\infty}$ & {$[4,4]$} \\
\hline$[4,3,6]$ & 3 edges & $D_{2} \times D_{\infty}($ twice $), \quad D_{4} \times D_{\infty}$ & {$[3,6]$} \\
\hline
\end{tabular}

4.3. Two ideal vertices. We have nine such Coxeter groups, namely the groups $[(3,5,3,6)],\left[\left(3,4^{3}\right)\right],[(3,4,3,6)],\left[\left(3^{3}, 6\right)\right],\left[3^{[3,3]}\right],\left[6,3^{1,1}\right],[3,6,3],[6,3,6]$, and $[4,4,4]$. Note that for these groups, we have only one edge segment in the fundamental domain to consider.

For the groups $\left[\left(3^{3}, 6\right)\right]$ and $[3,6,3]$, the edge extends to one of the non-compact edges, and hence we again have that there are no periodic geodesics of type I. So in both of these cases, we have that the maximal virtually infinite $\mathcal{V} C$ subgroups of hyperbolic type are isomorphic to $\mathbb{Z}, D_{\infty}, \mathbb{Z}_{2} \times \mathbb{Z}$, or $\mathbb{Z}_{2} \times D_{\infty}$. 
In the remaining cases, the edge reflects at both of its endpoints. The stabilizers we obtain, as well as the two cusp subgroups, are listed out in the following table:

Table 4. Structure of $\mathcal{V} \mathcal{C}$ subgroups of 2-ideal vertex groups.

\begin{tabular}{|c|c|c|}
\hline$\Gamma$ & $\operatorname{Stab}_{\Gamma}$ & Cusp \\
\hline$[(3,5,3,6)]$ & $D_{5} \times D_{\infty}$ & {$[3,6]$ (twice) } \\
\hline$\left[\left(3,4^{3}\right)\right]$ & $D_{3} \times D_{\infty}$ & {$[4,4]$ (twice) } \\
\hline$[(3,4,3,6)]$ & $D_{4} \times D_{\infty}$ & {$[3,6]$ (twice) } \\
\hline$\left[3^{[3,3]}\right]$ & $D_{4} *_{D_{2}} D_{4}$ & {$\left[3^{[3]}\right]$ (twice) } \\
\hline$\left[6,3^{1,1}\right]$ & $\left(D_{2} \times \mathbb{Z}_{2}\right) *_{D_{2}} D_{4}$ & {$[3,6]$ (twice) } \\
\hline$[6,3,6]$ & $D_{3} \times D_{\infty}$ & {$[3,6]$ (twice) } \\
\hline$[4,4,4]$ & $D_{2} \times D_{\infty}$ & {$[4,4]$ (twice) } \\
\hline
\end{tabular}

4.4. Three ideal vertices. We have two such Coxeter group: $\left[6,3^{[3]}\right]$ and $\left[4^{1,1,1}\right]$. Again, there will be no periodic geodesics of type I. So we obtain that the maximal virtually infinite $\mathcal{V} C$ subgroups of hyperbolic type are isomorphic to $\mathbb{Z}, D_{\infty}, \mathbb{Z}_{2} \times \mathbb{Z}$, or $\mathbb{Z}_{2} \times D_{\infty}$.

4.5. Four ideal vertices. There are three such Coxeter groups, namely the groups $\left[3^{[] \times[]}\right],\left[4^{[4]}\right]$ and $\left[(3,6)^{[2]}\right]$. It is clear that these groups have no periodic geodesics of type $I$, and hence the only maximal virtually infinite $\mathcal{V} C$ subgroups of hyperbolic type in both of these groups are isomorphic to $\mathbb{Z}, D_{\infty}, \mathbb{Z}_{2} \times \mathbb{Z}$, or $\mathbb{Z}_{2} \times D_{\infty}$.

\section{The algebraic $K$-theory of cell stabilizers in $E_{\mathscr{F} \mathcal{I N}}$}

In this section, we focus on finding the algebraic $K$-theory of the finite subgroups that occur as cell stabilizers for the $\Gamma$-action on $\mathbb{H}^{3}$, when $\Gamma$ ranges over the 32 groups we are studying. Recall from the discussion in Section 2 that these cell stabilizers will be (up to conjugacy) precisely the finite special subgroups. Since these can be read off from the Coxeter diagrams of the groups, one can easily see that, up to isomorphism, these groups are the following:

Finite special subgroups. $1, \mathbb{Z} / 2, D_{n}$ for $n=2,3,4,5,6,10$ (here $D_{n}$ denotes the dihedral group of order $2 n$ ), $D_{2} \times \mathbb{Z} / 2, D_{4} \times \mathbb{Z} / 2, D_{6} \times \mathbb{Z} / 2, S_{4}, S_{4} \times \mathbb{Z} / 2$, and $A_{5} \times \mathbb{Z} / 2$.

In the spectral sequence computing the homology $H_{*}^{\Gamma}\left(E_{\mathcal{F} \mathcal{I N}}(\Gamma) ; \mathbb{K} \mathbb{Z}^{-\infty}\right)$, the $E^{2}$-term is computed from the algebraic $K$-groups of the various cell stabilizers. 
So we need the algebraic $K$-groups for all of the finite groups appearing in the list above. For the convenience of the reader, we provide in Table 5 below the results of the computations in this section. Table 5 provides a list of all the non-trivial $K$-groups that occur amongst the finite groups we are considering.

Table 5. Lower algebraic $K$-theory of cell stabilizers in $E_{\mathcal{F} \mathcal{I N}}$.

\begin{tabular}{|c|c|c|c|}
\hline$F \in \mathcal{F} \mathcal{I} \mathcal{N}$ & $\mathrm{Wh}_{q} \neq 0, q \leq-1$ & $\widetilde{K}_{0} \neq 0$ & $\mathrm{Wh} \neq 0$ \\
\hline \hline$D_{2} \times \mathbb{Z} / 2$ & & $\mathbb{Z} / 2$ & \\
\hline$D_{5}$ & & & $\mathbb{Z}$ \\
\hline$D_{6}$ & $K_{-1} \cong \mathbb{Z}$ & & \\
\hline$D_{4} \times \mathbb{Z} / 2$ & & $\mathbb{Z} / 4$ & \\
\hline$D_{10}$ & $K_{-1} \cong \mathbb{Z}$ & & $\mathbb{Z}^{2}$ \\
\hline$D_{6} \times \mathbb{Z} / 2$ & $K_{-1} \cong \mathbb{Z}^{3}$ & $(\mathbb{Z} / 2)^{2}$ & \\
\hline$S_{4} \times \mathbb{Z} / 2$ & $K_{-1} \cong \mathbb{Z}$ & $\mathbb{Z} / 4$ & \\
\hline$A_{5} \times \mathbb{Z} / 2$ & $K_{-1} \cong \mathbb{Z}^{2}$ & $\mathbb{Z} / 2$ & $\mathbb{Z}^{2}$ \\
\hline
\end{tabular}

We now proceed to justify the results summarized in Table 5. It is well known that for $G$ a finite group $K_{q}(\mathbb{Z} G)$ is trivial for all $q \leq-2$ (see [C80a]), so we will focus exclusively on the functors $K_{-1}, \widetilde{K}_{0}$, and Wh. Next we point out that, for all but four of the finite groups in our list, their lower algebraic $K$-theory is well known. The relevant references are listed below for each of these groups.

- $\mathbb{Z} / 2$ : For the negative $K$-groups, we refer the reader to [C80a]; the fact that $K_{-1}(\mathbb{Z}[\mathbb{Z} / 2])=0$ can also be found in [Bas68, Theorem 10.6, pg. 695]. The vanishing of $\tilde{K}_{0}$ can be found in [CuR87, Corollary 5.17]. For information about the vanishing of Wh we refer the reader to [O89].

- $D_{2} \times \mathbb{Z} / 2$ : For the negative $K$-groups, we refer the reader to [C80a]. The formula in Bass [Bas68, Chapter 12] shows also that $K_{-1}\left(\mathbb{Z}\left[D_{2} \times \mathbb{Z} / 2\right]\right)=0$. For the information concerning Wh we refer the reader to [Ma78], [Ma80], [O89]. For the $\widetilde{K}_{0}$ see Remark 5.1.

- $D_{n}, n=2,3,4$ : For the vanishing of the negative $K$-groups, we refer the reader to [C80a]. For the $\widetilde{K}_{0}$ we refer the reader to [Re76]. In particular, the vanishing of $\widetilde{K}_{0}(\mathbb{Z} G)$ is proven for $G=D_{3}$ in [Re76, Theorem 8.2] and for $G=D_{4}$ in 
[Re76, Theorem 6.4]. For information about Wh we refer the reader to [Ma78], [Ma80] and [O89].

- $D_{5}$ : As far as we know the only $K$-groups found in the literature are $\widetilde{K}_{0}\left(\mathbb{Z} D_{5}\right) \cong 0$ (see [RU74, (2.6), pg. 506]) and $K_{q}\left(\mathbb{Z} D_{5}\right) \cong 0$ for all $q \leq-2$ (see [C80a]). To compute $K_{-1}\left(\mathbb{Z} D_{5}\right)$, we used results that can be found in [C80a], and [C80b], and to compute $\mathrm{Wh}\left(D_{5}\right)$, we used results that can be found in [Ma78], [Ma80] and [O89] (see the details in the Section 5.2).

- $D_{10}$ : As far as we know the only $K$-groups found in the literature are $\widetilde{K}_{0}\left(\mathbb{Z} D_{10}\right) \cong 0$ (by a straightforward modification of the argument in [RU74, Theorem 2.9]) and $K_{q}\left(\mathbb{Z} D_{10}\right)=0$ for all $q \leq-2$ (see [C80a]). For the $K_{-1}\left(\mathbb{Z} D_{10}\right)$, we used the results found in [C80a], and [C80b], and for $\mathrm{Wh}\left(D_{10}\right)$, we used results that can be found in [Ma78], [Ma80] and [O89] (see the details in the Section 5.3).

- $D_{6}$ : See the discussion in Section 5.1. The whitehead groups $\mathrm{Wh}_{q}\left(D_{6}\right)$ for $q \leq 1$ can also be found in [Pe98, Section 3], and [Or04, Section 5].

- $D_{4} \times \mathbb{Z} / 2$ : Ortiz in [Or04, Section 5] using results from [C80a] [C80b], [CuR87], [O89] and [Ma06] showed that $K_{q}\left(\mathbb{Z}\left[D_{4} \times \mathbb{Z} / 2\right]\right)=0, q \leq-1, \widetilde{K}_{0}\left(\mathbb{Z}\left[D_{4} \times\right.\right.$ $\mathbb{Z} / 2]) \cong \mathbb{Z} / 4$, and that $\mathrm{Wh}\left(D_{4} \times \mathbb{Z} / 2\right)$ is trivial.

- $S_{4}$ : Reiner and Ullom in [RU74, Theorem 3.2]) proved $\widetilde{K}_{0}\left(\mathbb{Z} S_{4}\right)$ is trivial. By [O89, Theorem 14.1], it follows that $\mathrm{Wh}\left(S_{4}\right)$ is trivial. By [C80b, Remark on pg. 606], it follows that $\widetilde{K}_{-1}\left(\mathbb{Z} S_{4}\right)$ is trivial.

- $S_{4} \times \mathbb{Z} / 2$ : By [C80a], it follows $\left.K_{q}\left(\mathbb{Z}\left[S_{4} \times \mathbb{Z} / 2\right]\right]\right)$ is trivial for all $q \leq-2$. Ortiz in $\left[\right.$ Or04, Section 5] showed $\left.K_{-1}\left(\mathbb{Z}\left[S_{4} \times \mathbb{Z} / 2\right]\right]\right) \cong \mathbb{Z}, \widetilde{K}_{0}\left(\mathbb{Z}\left[S_{4} \times \mathbb{Z} / 2\right]\right) \cong \mathbb{Z} / 4$, and $\mathrm{Wh}\left(S_{4} \times \mathbb{Z} / 2\right)$ is trivial.

For the remaining groups in our list, we detail the computations in the next few subsections.

5.1. The lower algebraic $\boldsymbol{K}$-theory of $\boldsymbol{D}_{6} \times \mathbb{Z} / 2$. To calculate $K_{-1}\left(\mathbb{Z}\left[D_{6} \times \mathbb{Z} / 2\right]\right)$, we use the following formula due to Carter [C80b, Theorem 3]. Let $G$ be a group of order $n$, let $p$ denote a prime number, let $\widehat{\mathbb{Z}}_{p}$ denote the $p$-adic integers and let $\widehat{\mathbb{Q}}_{p}$ denote the $p$-adic numbers. Then the following sequence is exact:

$0 \rightarrow K_{0}(\mathbb{Z}) \rightarrow K_{0}(\mathbb{Q} G) \oplus \bigoplus_{p \mid n} K_{0}\left(\hat{\mathbb{Z}}_{p} G\right) \rightarrow \bigoplus_{p \mid n} K_{0}\left(\hat{\mathbb{Q}}_{p} G\right) \rightarrow K_{-1}(\mathbb{Z} G) \rightarrow 0$

Since the group algebra $\mathbb{Q} D_{6} \cong \mathbb{Q}^{4} \times\left(M_{2}(\mathbb{Q})\right)^{2}$ (see [Or04, pg. 350]), it follows that the group algebra $\mathbb{Q}\left[D_{6} \times \mathbb{Z} / 2\right]$ is isomorphic to $\mathbb{Q}^{8} \times\left(M_{2}(\mathbb{Q})\right)^{4}$, and the same 
statement is true if $\mathbb{Q}$ is replaced by $\widehat{\mathbb{Q}}_{2}$ and $\widehat{\mathbb{Q}}_{3}$. Hence $K_{0}\left(\mathbb{Q}\left[D_{6} \times \mathbb{Z} / 2\right]\right) \cong$ $K_{0}\left(\widehat{\mathbb{Q}}_{2}\left[D_{6} \times \mathbb{Z} / 2\right]\right) \cong K_{0}\left(\widehat{\mathbb{Q}}_{3}\left[D_{6} \times \mathbb{Z} / 2\right]\right) \cong \mathbb{Z}^{12}$. The integral $p$-adic terms are $K_{0}\left(\widehat{\mathbb{Z}}_{2}\left[D_{6} \times \mathbb{Z} / 2\right]\right) \cong K_{0}\left(\mathbb{F}_{2}\left[D_{6} \times \mathbb{Z} / 2\right] \cong K_{0}\left(\mathbb{F}_{2}\left[D_{6}\right]\right) \cong \mathbb{Z}^{2}\right.$ (see [Or04,pg. 350]), and $K_{0}\left(\widehat{\mathbb{Z}}_{3}\left[D_{6} \times \mathbb{Z} / 2\right]\right) \cong K_{0}\left(\mathbb{F}_{3}\left[D_{6} \times \mathbb{Z} / 2\right]\right) \cong K_{0}\left(\mathbb{F}_{3}\left[(\mathbb{Z} / 2)^{3}\right]\right) \cong \mathbb{Z}^{8}$. Carter also shows in [C80a] that $K_{-1}\left(\mathbb{Z}\left[D_{6} \times \mathbb{Z} / 2\right]\right)$ is torsion free, so counting ranks in the exact sequence, we have that $K_{-1}\left(\mathbb{Z}\left[D_{6} \times \mathbb{Z} / 2\right]\right) \cong \mathbb{Z}^{3}$.

To compute $\widetilde{K}_{0}\left(\mathbb{Z}\left[D_{6} \times \mathbb{Z} / 2\right]\right)$, consider the following Cartesian square

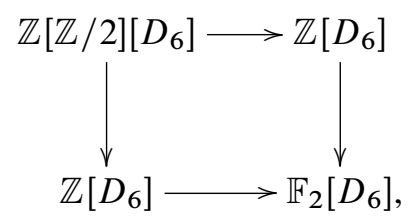

which yields the Mayer-Vietories sequence (see [CuR87, Theorem 49.27])

$$
\begin{aligned}
K_{1}\left(\mathbb{Z}\left[D_{6} \times \mathbb{Z} / 2\right]\right) \longrightarrow K_{1}\left(\mathbb{Z} D_{6}\right) \oplus K_{1}\left(\mathbb{Z} D_{6}\right) \stackrel{\varphi}{\longrightarrow} K_{1}\left(\mathbb{F}_{2}\left[D_{6}\right]\right) \\
\longrightarrow \widetilde{K}_{0}\left(\mathbb{Z}[\mathbb{Z} / 2]\left[D_{6}\right]\right) \longrightarrow \widetilde{K}_{0}\left(\mathbb{Z} D_{6}\right) \oplus \widetilde{K}_{0}\left(\mathbb{Z} D_{6}\right) \longrightarrow 0 .
\end{aligned}
$$

We now proceed to compute the various terms appearing in this sequence.

We start by looking at the terms involving $\mathbb{Z} D_{6}$. In [Re76] Reiner shows that $\widetilde{K}_{0}\left(\mathbb{Z} D_{6}\right)$ is trivial. $K_{1}\left(\mathbb{Z} D_{6}\right)$ can be computed as follows: since $\mathrm{Wh}(G)$ equals $K_{1}(\mathbb{Z} G) /\left\{ \pm G^{a b}\right\}$, the rank of $K_{1}(\mathbb{Z} G)$ is equal to the rank of $\mathrm{Wh}(G)$. But the rank of $\mathrm{Wh}(G)$ is $y=r-q$, where $r$ denotes the number of irreducible real representations of $G$, and $q$ denotes the number of irreducible rational representations of $G$. In [Bas65] Bass shows that $r$ is equal to the number of conjugacy classes of sets $\left\{x, x^{-1}\right\}, x \in G$, and $q$ is the number of conjugacy classes of cyclic subgroups of $G$ (see also [Mi66]). For $G=D_{6}$, a direct calculations shows that $r=q$, and hence that $\operatorname{Wh}(G)$ is purely torsion. Next note that the torsion part of $K_{1}(\mathbb{Z} G)$ is $\{ \pm 1\} \oplus G^{a b} \oplus S K_{1}(\mathbb{Z} G)$ (see [W74]), and hence we have that $\mathrm{Wh}\left(D_{6}\right)=S K_{1}\left(\mathbb{Z} D_{6}\right)$. Since Magurn [Ma78] has shown that $S K_{1}\left(\mathbb{Z} D_{6}\right)$ is trivial, we see that $\mathrm{Wh}\left(D_{6}\right)$ is trivial. Since $\left(D_{6}\right)^{a b}=$ $(\mathbb{Z} / 2)^{2}$, we obtain that $K_{1}\left(\mathbb{Z}\left[D_{6}\right]\right)=(\mathbb{Z} / 2)^{3}$.

Next we consider the remaining terms in the Mayer-Vietoris sequence. For $G=$ $D_{6} \times \mathbb{Z} / 2$, Magurn in [Ma80, Corollary 11] shows that $\mathrm{Wh}\left(D_{6} \times \mathbb{Z} / 2\right)=0$ (note that the rank of $\mathrm{Wh}(G \times \mathbb{Z} / 2)$ is twice the rank of $\mathrm{Wh}(G)$ since $r$ and $q$ get doubled, see Sections 5.2 and 5.3). Since $\left(D_{6} \times \mathbb{Z} / 2\right)^{a b}=(\mathbb{Z} / 2)^{3}$, this yields $K_{1}\left(\mathbb{Z}\left[D_{6} \times \mathbb{Z} / 2\right]\right)=$ $(\mathbb{Z} / 2)^{4}$. Finally, Magurn in [Ma06, Example 9]) shows that $K_{1}\left(\mathbb{F}_{2}\left[D_{6}\right]\right)=(\mathbb{Z} / 2)^{4}$. Substituting all the known terms into the exact sequence in (1) yields the following exact sequence:

$$
(\mathbb{Z} / 2)^{4} \stackrel{\sigma}{\longrightarrow}(\mathbb{Z} / 2)^{3} \oplus(\mathbb{Z} / 2)^{3} \stackrel{\varphi}{\longrightarrow}(\mathbb{Z} / 2)^{4} \longrightarrow \widetilde{K}_{0}\left(\mathbb{Z}[\mathbb{Z} / 2]\left[D_{6}\right]\right) \longrightarrow 0 .
$$

Next, we study the image of $\varphi: K_{1}\left(\mathbb{Z} D_{6}\right) \oplus K_{1}\left(\mathbb{Z} D_{6}\right) \rightarrow K_{1}\left(\mathbb{F}_{2}\left[D_{6}\right]\right)$. We claim that $\operatorname{im}(\varphi)=(\mathbb{Z} / 2)^{2}$. This can be seen as follows: first $\operatorname{im}(\varphi)=\operatorname{im}(\psi)$ 
where $\psi: K_{1}\left(\mathbb{Z} D_{6}\right) \rightarrow K_{1}\left(\mathbb{F}_{2}\left[D_{6}\right]\right)$ is induced by the canonical ring homomorphism $\mathbb{Z} \rightarrow \mathbb{F}_{2}$. Note the $K_{1}(\mathbb{Z})$ is a direct summand of $K_{1}\left(\mathbb{Z} D_{6}\right)$ isomorphic to $\mathbb{Z} / 2$; but this summand goes to zero in $K_{1}\left(\mathbb{F}_{2}\left[D_{6}\right]\right)$ since it factors through the following commutative square:

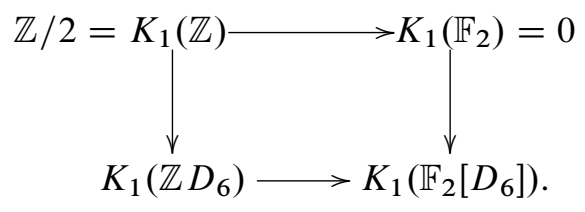

Since $K_{1}\left(\mathbb{Z} D_{6}\right)=(\mathbb{Z} / 2)^{3}$, this forces $\operatorname{dim}_{\mathbb{F}_{2}}(\operatorname{im}(\varphi)) \leq 2$. Now from the exact sequences given in (1) and (2), we have that

$$
\operatorname{dim}_{\mathbb{F}_{2}}(\operatorname{im}(\varphi))=2 \operatorname{dim}_{\mathbb{F}_{2}}\left(K_{1}\left(\mathbb{Z}\left[D_{6}\right]\right)\right)-\operatorname{dim}_{\mathbb{F}_{2}}(\operatorname{ker}(\varphi))=6-\operatorname{dim}_{\mathbb{F}_{2}}(\operatorname{im}(\sigma)) .
$$

Since $\operatorname{dim}_{\mathbb{F}_{2}}(\operatorname{im}(\sigma)) \leq 4$, we see that $\operatorname{dim}_{\mathbb{F}_{2}}(\operatorname{im}(\varphi)) \geq 2$, which forces $\operatorname{im}(\varphi) \cong$ $(\mathbb{Z} / 2)^{2}$. The exact sequence now yields $\widetilde{K}_{0}\left(\mathbb{Z}[\mathbb{Z} / 2]\left[D_{6}\right]\right) \cong(\mathbb{Z} / 2)^{2}$.

Remark. In a preliminary version of this paper, the authors incorrectly stated that $\widetilde{K}_{0}\left(\mathbb{Z}\left[D_{2} \times \mathbb{Z} / 2\right]\right)$ vanished. The second author realized, while working on a new project (see [FO]), that this group is in fact non-trivial, as is proved in [EH79, pg. 161]. Using the same argument as in the case of $D_{6} \times \mathbb{Z} / 2$ above, one can show that $\widetilde{K}_{0}\left(\mathbb{Z}\left[D_{2} \times \mathbb{Z} / 2\right]\right) \cong \mathbb{Z} / 2$.

In one of our earlier papers (see [LO07, pg. 542]), the incorrect vanishing of $\widetilde{K}_{0}\left(\mathbb{Z}\left[D_{2} \times \mathbb{Z} / 2\right]\right)$ was used, leading to an incorrect computation for the $\widetilde{K}_{0}$ of $Q=\left(D_{2} \times \mathbb{Z} / 2\right) * D_{2}\left(D_{2} \times \mathbb{Z} / 2\right)$. We would like to use this opportunity to state the correct computation of this group: $\widetilde{K}_{0}(\mathbb{Z} Q) \cong(\mathbb{Z} / 2)^{2} \oplus N K_{0}\left(\mathbb{Z} D_{2} ; B_{1}, B_{2}\right)$, where $B_{i}=\mathbb{Z}\left[\left(D_{2} \times Z / 2\right) \backslash D_{2}\right]$ is the $\mathbb{Z} D_{2}$-bimodule generated by $\left(D_{2} \times \mathbb{Z} / 2\right) \backslash$ $D_{2}$ for $i=1,2$. However, as was shown in [LO07, Theorem 5.2], the Nil-group $N K_{0}\left(\mathbb{Z} D_{2} ; B_{1}, B_{2}\right)$ is isomorphic to a countably infinite direct sum $\bigoplus_{\infty} \mathbb{Z} / 2$ of cyclic groups of order two, and hence the abstract isomorphism type of the group $\widetilde{K}_{0}(\mathbb{Z} Q)$ is correct as stated in [LO07]. In particular, the computation presented in the main theorem [LO07, Theorem 1.1] remains correct as stated.

5.2. The computation of the $K$-groups $K_{-1}\left(\mathbb{Z} D_{5}\right)$, and $W h\left(D_{5}\right)$. To compute $K_{-1}\left(\mathbb{Z} D_{5}\right)$, we need Carter's formula for $K_{-1}$, [C80b, Theorem 3], the reader is referred to Section 5.1.

$$
\begin{aligned}
0 \longrightarrow K_{0}(\mathbb{Z}) \longrightarrow K_{0}\left(\mathbb{Q} D_{5}\right) \oplus \bigoplus_{p \mid n} K_{0}\left(\widehat{\mathbb{Z}}_{p} D_{5}\right) \\
\longrightarrow \bigoplus_{p \mid n} K_{0}\left(\widehat{\mathbb{Q}}_{p} D_{5}\right) \longrightarrow K_{-1}\left(\mathbb{Z} D_{5}\right) \longrightarrow 0
\end{aligned}
$$


The group algebra $\mathbb{Q} D_{5}$ is isomorphic to $\mathbb{Q}^{2} \times M_{2}(\mathbb{Q}[\sqrt{5}]$ ) (see [Se77, pgs. 36$38]$ ), and the same statement is true if $\mathbb{Q}$ is replaced by $\widehat{\mathbb{Q}}_{2}$ (recall that $\sqrt{5} \notin \widehat{\mathbb{Q}}_{2}$ ). For $p=5$, the group algebra $\widehat{\mathbb{Q}}_{5} D_{5}$ is isomorphic to $\left(\widehat{\mathbb{Q}}_{5}\right)^{2} \times M_{2}\left(\widehat{\mathbb{Q}}_{5}\right)$. Hence $K_{0}\left(\widehat{\mathbb{Q}}_{2}\left[D_{5}\right]\right) \cong K_{0}\left(\mathbb{Q}\left[D_{5}\right]\right) \cong K_{0}\left(\widehat{\mathbb{Q}}_{5}\left[D_{5}\right]\right) \cong \mathbb{Z}^{3}$. By using techniques described in [CuR81, Section 5], we have that $K_{0}\left(\widehat{\mathbb{Z}}_{5}\left[D_{5}\right]\right) \cong K_{0}\left(\mathbb{F}_{5}\left[D_{5}\right]\right) \cong K_{0}\left(\mathbb{F}_{5}[\mathbb{Z} / 2]\right)=$ $K_{0}\left(\mathbb{F}_{5} \times \mathbb{F}_{5}\right)=\mathbb{Z}^{2}$. Similarly, we have that $K_{0}\left(\widehat{\mathbb{Z}}_{2}\left[D_{5}\right]\right) \cong K_{0}\left(\mathbb{F}_{2}\left[D_{5}\right]\right) \cong K_{0}\left(\mathbb{F}_{2} \times\right.$ $\left.M_{2}\left(\mathbb{F}_{2}\right)\right)=\mathbb{Z}^{2}$. Carter also shows in [C80a] that $K_{-1}\left(\mathbb{Z}\left[D_{5}\right]\right)$ is torsion free, so counting ranks as before, we obtain $K_{-1}\left(\mathbb{Z} D_{5}\right) \cong 0$.

Next, we compute $\operatorname{Wh}\left(D_{5}\right)$. Recall that $\operatorname{Wh}(G)=\mathbb{Z}^{y} \oplus S K_{1}(\mathbb{Z} G)$. Magurn in [Ma78] proves that $S K_{1}$ vanishes for all finite dihedral groups. The rank of the torsion free part is $y=r-q$ (see Section 5.1). For the group $D_{5}$, a direct calculation shows that $r=4$ and $q=3$, yielding $\mathrm{Wh}\left(D_{5}\right) \cong \mathbb{Z}^{r-q} \cong \mathbb{Z}$.

5.3. The computation of the $K$-groups $K_{-1}\left(\mathbb{Z} D_{10}\right)$, and $W h\left(D_{10}\right)$. To calculate $K_{-1}\left(\mathbb{Z} D_{10}\right)$, again using Carter's formula for $K_{-1}[\mathrm{C} 80 \mathrm{~b}$, Theorem 3], we have (see Sections 5.1 and 5.2)

$$
\begin{aligned}
0 \longrightarrow & K_{0}(\mathbb{Z}) \longrightarrow K_{0}\left(\mathbb{Q} D_{10}\right) \oplus \bigoplus_{p \mid n} K_{0}\left(\widehat{\mathbb{Z}}_{p} D_{10}\right) \\
& \longrightarrow \bigoplus_{p \mid n} K_{0}\left(\widehat{\mathbb{Q}}_{p} D_{10}\right) \longrightarrow K_{-1}\left(\mathbb{Z} D_{10}\right) \longrightarrow 0
\end{aligned}
$$

The group algebra $\mathbb{Q}\left[D_{5} \times \mathbb{Z} / 2\right]$ is isomorphic to $\mathbb{Q}^{4} \times\left(M_{2}(\mathbb{Q}[\sqrt{5}])\right)^{2}$ (see Section 5.2) and the same statement is true if $\mathbb{Q}$ is replaced by $\widehat{\mathbb{Q}}_{2}$. For $p=5$, we have isomorphisms of algebras $\widehat{\mathbb{Q}}_{5}\left[D_{5} \times \mathbb{Z} / 2\right] \cong\left(\widehat{\mathbb{Q}}_{5}\right)^{4} \times\left(M_{2}\left(\widehat{\mathbb{Q}}_{5}\right)\right)^{2}$. Hence $K_{0}\left(\mathbb{Q}\left[D_{5} \times \mathbb{Z} / 2\right]\right) \cong K_{0}\left(\widehat{\mathbb{Q}}_{2}\left[D_{5} \times \mathbb{Z} / 2\right]\right) \cong K_{0}\left(\widehat{\mathbb{Q}}_{5}\left[D_{5} \times \mathbb{Z} / 2\right]\right) \cong \mathbb{Z}^{6}$. The integral $p$-adic terms are $K_{0}\left(\widehat{\mathbb{Z}}_{2}\left[D_{5} \times \mathbb{Z} / 2\right]\right) \cong K_{0}\left(\mathbb{F}_{2}\left[D_{5} \times \mathbb{Z} / 2\right]\right) \cong K_{0}\left(\mathbb{F}_{2}\left[D_{5}\right]\right) \cong \mathbb{Z}^{2}$ (see Section 5.2), and $K_{0}\left(\widehat{\mathbb{Z}}_{5}\left[D_{5} \times \mathbb{Z} / 2\right]\right) \cong K_{0}\left(\mathbb{F}_{5}\left[D_{5} \times \mathbb{Z} / 2\right]\right) \cong K_{0}\left(\mathbb{F}_{5}\left[(\mathbb{Z} / 2)^{2}\right]\right) \cong$ $\mathbb{Z}^{4}$. Carter also shows in $[C 80 \mathrm{a}]$ that $K_{-1}\left(\mathbb{Z}\left[D_{10}\right]\right)$ is torsion free, so counting ranks in the exact sequence, we have that $K_{-1}\left(\mathbb{Z} D_{10}\right) \cong \mathbb{Z}$.

Next, we compute $\mathrm{Wh}\left(D_{10}\right)$. Magurn in [Ma78] proves that $S K_{1}$ vanishes for all finite dihedral groups, then as before $\operatorname{Wh}\left(D_{10}\right) \cong \mathbb{Z}^{y}$. Since $\operatorname{rk}(\mathrm{Wh}(G \times \mathbb{Z} / 2))=$ $2 \operatorname{rk}(\mathrm{Wh}(G))$ and $\mathrm{Wh}\left(D_{5}\right) \cong \mathbb{Z}$, it follows that $\mathrm{Wh}\left(D_{10}\right) \cong \mathbb{Z}^{2}$.

5.4. The lower algebraic $K$-theory of $A_{5} \times \mathbb{Z} / 2$. To compute $\mathrm{Wh}_{q}\left(A_{5} \times \mathbb{Z} / 2\right)$ for $q \leq 1$, we first claim that

$$
\mathrm{Wh}_{q}\left(A_{5}\right)= \begin{cases}\mathbb{Z}, & q=1 \\ 0, & q \leq 0\end{cases}
$$

This can be seen as follows. By [O89, Theorem 14.6], we have that $S K_{1}\left(\mathbb{Z} A_{5}\right)=0$. The group $A_{5}$ has precisely five (mutually nonisomorphic) irreducible real represen- 
tation, giving $r=5$. In $A_{5}$ the conjugacy classes of cyclic subgroups are represented by the trivial subgroup $\{e\},\langle(12)(34)\rangle,\langle(123)\rangle,\langle(12345)\rangle$ giving us that $q=4$. This forces $\mathrm{Wh}\left(A_{5}\right) \cong \mathbb{Z}^{r-q}=\mathbb{Z}$. By [RU74, Theorem 3.2], we have that $\widetilde{K}_{0}\left(\mathbb{Z} A_{5}\right)=0$; Dress induction as used in [O89, Theorem 11.2] shows that $K_{-1}\left(\mathbb{Z} A_{5}\right)=0$, and by [C80a] we have that $K_{q}\left(\mathbb{Z} A_{5}\right)=0$ for $q \leq-2$.

Now let us compute $\mathrm{Wh}\left(A_{5} \times \mathbb{Z} / 2\right)$. Magurn in [Ma07, Example 5] shows that $S K_{1}\left(\mathbb{Z}\left[A_{5} \times \mathbb{Z} / 2\right]\right)=0$. Since $\operatorname{rk}(\mathrm{Wh}(G \times \mathbb{Z} / 2))=2 \operatorname{rk}(\mathrm{Wh}(G))$ and $\mathrm{Wh}\left(A_{5}\right) \cong \mathbb{Z}$ we get $\mathrm{Wh}\left(A_{5} \times \mathbb{Z} / 2\right) \cong \mathbb{Z}^{2}$.

Next, we claim $\widetilde{K}_{0}\left(\mathbb{Z}\left[A_{5} \times \mathbb{Z} / 2\right]\right) \cong \mathbb{Z} / 2$. To see this, let $H$ be a subgroup of $G$. For any locally free $\mathbb{Z} G$-module $M$ its restriction to $H$ (denoted by $M_{H}$ ) is a locally free $\mathbb{Z} H$-module. The mapping defined by $[M] \rightarrow\left[M_{H}\right]$ gives a homomorphism of $\widetilde{K}_{0}(\mathbb{Z} G) \rightarrow \widetilde{K}_{0}(\mathbb{Z} H)$.

A group $H$ is hyper-elementary if $H$ is a semidirect product $N \rtimes P$ of a cyclic normal subgroup $N$ and a subgroup $P$ of prime order, where $(|N|,|P|)=1$. Let $\mathscr{H}(G)$ consist of one representative from each conjugacy class of hyper-elementary subgroups of $G$. We shall need the following result presented by Reiner and Ullom in [RU74, Theorem 3.1]: for every finite group $G$, the map

$$
\tilde{K}_{0}(\mathbb{Z} G) \rightarrow \prod_{H \in \mathscr{H}(G)} \tilde{K}_{0}(\mathbb{Z} H)
$$

is a monomorphism. Observe that $\mathscr{H}\left(A_{5}\right)$ consists of subgroups of $A_{5}$ isomorphic to one of $D_{2}, D_{3}$ and $D_{5}$. Note that the hyper-elementary subgroups of $G \times \mathbb{Z} / 2$ are of the form $H$ or $H \times \mathbb{Z} / 2$ for $H \in \mathscr{H}(G)$. In particular, the hyper-elementary subgroups of $A_{5} \times \mathbb{Z} / 2$ are all isomorphic to one of: $D_{2}, D_{3}$ and $D_{5}, D_{2} \times \mathbb{Z} / 2$, $D_{3} \times \mathbb{Z} / 2 \cong D_{6}$, and $D_{5} \times \mathbb{Z} / 2 \cong D_{10}$. By the results already mentioned in Sections 5.2, 5.3, and the remark at the end of Section 5.1, we have $\widetilde{K}_{0}(\mathbb{Z} H)=0$ for all $H \in \mathscr{H}\left(A_{5} \times \mathbb{Z} / 2\right)$ except for $H=D_{2} \times \mathbb{Z} / 2$, where $\widetilde{K}_{0}(\mathbb{Z} H) \cong \mathbb{Z} / 2$. This implies that the target of the map given in (3) is isomorphic to $\mathbb{Z} / 2$, and injectivity of the map now gives us an injection $\widetilde{K}_{0}\left(\mathbb{Z}\left[A_{5} \times \mathbb{Z} / 2\right]\right) \hookrightarrow \mathbb{Z} / 2$. Since it is known that $\widetilde{K}_{0}\left(\mathbb{Z}\left[A_{5} \times \mathbb{Z} / 2\right]\right.$ ) is non trivial (see [EH79, Theorem on pg. 161]), it follows that $\widetilde{K}_{0}\left(\mathbb{Z}\left[A_{5} \times \mathbb{Z} / 2\right]\right) \cong \mathbb{Z} / 2$, as desired.

Finally, using results from [C80a], Magurn showed that $K_{-1}\left(\mathbb{Z}\left[A_{5} \times \mathbb{Z} / 2\right]\right) \cong \mathbb{Z}^{2}$. The explicit computation of this $K$-group can be found in [LMO, Section 3.2].

\section{Cokernels of relative assembly maps for maximal infinite virtually cyclic subgroups}

In view of Corollary 3.4, we will need for our computations the cokernels of the relative assembly maps for the various maximal infinite virtually cyclic subgroups of type I. From the tables 2, 3, 4 computed in Section 4, we have the following list 
containing all the maximal infinite virtually cyclic subgroups that appear in the 32 groups we are interested in:

Maximal infinite virtually cyclic subgroups. $\mathbb{Z}, D_{\infty}, \mathbb{Z} \times \mathbb{Z} / 2, D_{\infty} \times \mathbb{Z} / 2, D_{4} * D_{2}$ $D_{4},\left(D_{2} \times \mathbb{Z} / 2\right) *_{D_{2}} D_{4}$, and $D_{n} \times D_{\infty}$ for $n=2,3,4,5$.

We first note that, for the groups in our list, the cokernels are known to be trivial in the following cases:

- $\mathbb{Z}$ : by work of Bass [Bas68].

- $D_{\infty}$ : by work of Waldhausen [Wd78].

- $\mathbb{Z} \times \mathbb{Z} / 2$, and $D_{\infty} \times \mathbb{Z} / 2$ : by work of Pearson [Pe98, Section 2].

- $D_{3} \times D_{\infty}$ : by work of the authors [LO07, Section 4]

Finally, the authors have also shown in [LO07, Section 4] that for the group $D_{2} \times D_{\infty}$, the cokernels of the relative assembly map for $n=0,1$ are countably infinite direct sums of $\mathbb{Z} / 2$. The remaining four groups in our list will be discussed in the following subsections.

Observe that by a result of Farrell and Jones [FJ95], the cokernels of the relative assembly maps $H_{n}^{V}\left(E_{\mathscr{F} \mathcal{I N}}(V) \rightarrow *\right)$ that we are interested in are automatically trivial for $n<-1$ (in fact, both the source and target groups vanish in this case). In the same paper, they establish that for the case $n=-1$, these cokernels are finitely generated, which by results of Farrell [F77], Ramos [Ra07], and Grunewald [G07], implies that the cokernel is actually trivial. In particular, we only need to focus on the cases $n=0$, and $n=1$. These cokernels are precisely the elusive Bass, Farrell, and Waldhausen Nil-groups. We are able to identify these cokernels exactly, with the exception of the case $D_{4} \times D_{\infty}$. For this group, we content ourselves with summarizing what we were able to obtain in Subsection 6.4. We summarize the non-trivial cokernels in Table 6.

Table 6. Cokernels of relative assembly map for maximal infinite $V \in \mathcal{V} C$.

\begin{tabular}{|c|c|c|}
\hline$V \in \mathcal{V C}$ & $H_{0}^{V}\left(E_{\mathcal{F} \mathcal{I N}}(V) \rightarrow *\right) \neq 0$ & $H_{1}^{V}\left(E_{\mathcal{F} \mathcal{N N}}(V) \rightarrow *\right) \neq 0$ \\
\hline \hline$D_{2} \times D_{\infty}$ & $\bigoplus_{\infty} \mathbb{Z} / 2$ & $\bigoplus_{\infty} \mathbb{Z} / 2$ \\
\hline$D_{4} * D_{2} D_{4}$ & $\bigoplus_{\infty} \mathbb{Z} / 2$ & $\bigoplus_{\infty} \mathbb{Z} / 2$ \\
\hline$\left(D_{2} \times \mathbb{Z} / 2\right) *_{D_{2}} D_{4}$ & $\bigoplus_{\infty} \mathbb{Z} / 2$ & $\bigoplus_{\infty} \mathbb{Z} / 2$ \\
\hline$D_{4} \times D_{\infty}$ & $\mathrm{Nil}_{0}$ & $\mathrm{Nil}_{1}$ \\
\hline
\end{tabular}


6.1. The lower algebraic $\boldsymbol{K}$-theory of $\boldsymbol{D}_{\mathbf{5}} \times \boldsymbol{D}_{\infty}$. First, note that $D_{5} \times D_{\infty} \cong$ $D_{10} *_{D_{5}} D_{10}$. As mentioned earlier $K_{n}(\mathbb{Z} Q)$ is zero for $n<-1$ (see [FJ95]). Since $K_{-1}\left(\mathbb{Z} D_{5}\right)=0$ (see Section 5.2), and $K_{-1}\left(\mathbb{Z} D_{10}\right)=\mathbb{Z}$ (see Section 5.3), we see that for $Q=D_{10} *_{D_{5}} D_{10}$, we have $K_{-1}(\mathbb{Z} Q)=\mathbb{Z} \oplus \mathbb{Z}$.

For the remaining $K$-groups, we make use of [CP02, Lemma 3.8]. This gives us that $\widetilde{K}_{0}(\mathbb{Z} Q) \cong N K_{0}\left(\mathbb{Z} D_{5} ; C_{1}, C_{2}\right)$, where $C_{i}=\mathbb{Z}\left[D_{10}-D_{5}\right]$ is the $\mathbb{Z} D_{5^{-}}$ bimodule generated by $D_{10}-D_{5}$ for $i=1$, 2, (see Sections 5.2 and 5.3 for the $\widetilde{K}_{0}\left(\mathbb{Z} D_{n}\right)$ for $\left.n=5,10\right)$, and $\mathrm{Wh}(Q) \cong \mathbb{Z}^{3} \oplus N K_{1}\left(\mathbb{Z} D_{5} ; C_{1}, C_{2}\right)$, with $C_{1}$ and $C_{2}$ as before, (see Sections 5.2 and 5.3 for the $\operatorname{Wh}\left(D_{n}\right)$ for $n=5,10$ ). The Nil-groups appearing in these computations are the Waldhausen Nil-groups.

Now by [LO08], we know that $N K_{i}\left(\mathbb{Z} D_{5} ; C_{1}, C_{2}\right)=0$ for $i=0,1$, vanishes if and only if the corresponding Farrell Nil-group vanishes for the canonical index two subgroup $D_{5} \times \mathbb{Z} \triangleleft D_{5} \times D_{\infty}$. Note that in this case, the Farrell Nil-group is untwisted, and hence is just the Bass Nil-group $N K_{i}\left(\mathbb{Z} D_{5}\right)$. But Harmon [Ha87] has shown that for finite groups $G$ of square-free order (such as $D_{5}$ ), the Bass Nil group $N K_{i}(\mathbb{Z} G)$ vanishes for $i=0,1$. We summarize our computations in the following:

$$
\mathrm{Wh}_{q}\left(D_{5} \times D_{\infty}\right)= \begin{cases}\mathbb{Z}^{3}, & q=1 \\ 0, & q=0 \\ \mathbb{Z}^{2}, & q=-1 \\ 0, & q \leq-2\end{cases}
$$

and both the cokernels of the relative assembly map vanish for this group.

6.2. The lower algebraic $K$-theory of $\left(D_{\mathbf{2}} \times \mathbb{Z} / \mathbf{2}\right) *_{\boldsymbol{D}_{\mathbf{2}}} \boldsymbol{D}_{\mathbf{4}}$. As before $K_{n}(\mathbb{Z} Q)$ is zero for $n<-1$ (see [FJ95]). Since $K_{-1}\left(\mathbb{Z} D_{2}\right)=0, K_{-1}\left(\mathbb{Z}\left[D_{2} \times \mathbb{Z} / 2\right]\right)=0$, and $K_{-1}\left(\mathbb{Z} D_{4}\right)=0$, we see that for $Q=\left(D_{2} \times \mathbb{Z} / 2\right) *_{D_{2}} D_{4}$, we have that $K_{-1}(\mathbb{Z} Q)=0$.

For the remaining $K$-groups, using [CP02, Lemma 3.8], we have that $\widetilde{K}_{0}(\mathbb{Z} Q) \cong$ $\mathbb{Z} / 2 \oplus N K_{0}\left(\mathbb{Z} D_{2} ; A_{1}, A_{2}\right)$, where $A_{1}=\mathbb{Z}\left[\left(D_{2} \times \mathbb{Z} / 2\right)-D_{2}\right]$ is the $\mathbb{Z} D_{2}$-bimodule generated by $\left(D_{2} \times \mathbb{Z} / 2\right)-D_{2}$, and $A_{2}=\mathbb{Z}\left[D_{4}-D_{2}\right]$ is the $\mathbb{Z} D_{2}$-bimodule generated by $D_{4}-D_{2}$. Similarly, we have that $\mathrm{Wh}(Q) \cong N K_{1}\left(\mathbb{Z} D_{2} ; A_{1}, A_{2}\right)$, where $A_{1}, A_{2}$ are the bimodules defined above.

Recall that in [LO07, Theorem 5.2], the authors established that (1) $\widetilde{K}_{0}\left(\mathbb{Z}\left[D_{2} \times\right.\right.$ $\left.\left.D_{\infty}\right]\right) \cong \bigoplus_{\infty} \mathbb{Z} / 2$ and (2) $\mathrm{Wh}\left(D_{2} \times D_{\infty}\right) \cong \bigoplus_{\infty} \mathbb{Z} / 2$. The computation reduced to showing that the Waldhausen Nil-groups $N K_{i}\left(\mathbb{Z} D_{2} ; A_{2}, A_{2}\right)$ is isomorphic to an infinite countable sum of $\mathbb{Z} / 2$ (where the bimodule $A_{2}$ is defined in the previous paragraph). This was achieved by establishing (1) the existence of an injection, and (2) the existence of a (different) surjection, from the Bass Nil-group $N K_{i}\left(\mathbb{Z} D_{2}\right) \cong$ $\bigoplus_{\infty} \mathbb{Z} / 2$ into the corresponding Waldhausen Nil-group $N K_{i}\left(\mathbb{Z} D_{2} ; A_{2}, A_{2}\right)$. But 
the reader can verify that the argument given in [LO07] applies verbatim to the Waldhausen Nil-groups $N K_{i}\left(\mathbb{Z} D_{2} ; A_{1}, A_{2}\right)$ appearing in our present computation.

We conclude that the lower algebraic $K$-theory of $\left(D_{2} \times \mathbb{Z} / 2\right) *_{D_{2}} D_{4}$ is given by

$$
\mathrm{Wh}_{q}\left(\left(D_{2} \times \mathbb{Z} / 2\right) *_{D_{2}} D_{4}\right)= \begin{cases}\bigoplus_{\infty} \mathbb{Z} / 2, & q=1, \\ \bigoplus_{\infty} \mathbb{Z} / 2, & q=0, \\ 0, & q \leq-1,\end{cases}
$$

and that the cokernels of the relative assembly map are both isomorphic to $\bigoplus_{\infty} \mathbb{Z} / 2$.

6.3. The lower algebraic $K$-theory of $D_{\mathbf{4}} \boldsymbol{*}_{\boldsymbol{D}_{\mathbf{2}}} \boldsymbol{D}_{\mathbf{4}}$. As before $K_{n}(\mathbb{Z} Q)$ is zero for $n<-1$ (see [FJ95]). Since $K_{-1}\left(\mathbb{Z} D_{2}\right)=0$ and $K_{-1}\left(\mathbb{Z} D_{4}\right)=0$, we see that for $Q=D_{4} *_{D_{2}} D_{4}$, we have that $K_{-1}(\mathbb{Z} Q)=0$.

For the remaining $K$-groups, using [CP02, Lemma 3.8], we have that $\widetilde{K}_{0}(\mathbb{Z} Q) \cong$ $N K_{0}\left(\mathbb{Z} D_{2} ; F_{1}, F_{2}\right)$, where for $i=1,2, F_{i}=\mathbb{Z}\left[D_{4}-D_{2}\right]$ is the $\mathbb{Z} D_{2}$-bimodule generated by $D_{4}-D_{2}$. Similarly, we have that $\mathrm{Wh}(Q) \cong N K_{1}\left(\mathbb{Z} D_{2} ; F_{1}, F_{2}\right)$, with $F_{1}$ and $F_{2}$ as before.

Now using [LO07, Theorem 5.2], we conclude that for $Q=D_{4} * D_{2} D_{4}$

$$
\mathrm{Wh}_{q}(Q)= \begin{cases}\bigoplus_{\infty} \mathbb{Z} / 2, & q=1 \\ \bigoplus_{\infty} \mathbb{Z} / 2, & q=0 \\ 0, & q \leq-1\end{cases}
$$

and that the cokernels of the relative assembly map are both isomorphic to $\bigoplus_{\infty} \mathbb{Z} / 2$.

6.4. The lower algebraic $\boldsymbol{K}$-theory of $\boldsymbol{D}_{\mathbf{4}} \times \boldsymbol{D}_{\infty}$. The authors were unable to obtain an explicit computation for this group. In this case, we have that $D_{4} \times D_{\infty} \cong$ $\left(D_{4} \times \mathbb{Z} / 2\right) *_{D_{4}}\left(D_{4} \times \mathbb{Z} / 2\right)$, and we are interested in the Waldhausen Nil-groups associated to this splitting. A special case of recent independent work of several authors (including Davis [D], Davis-Khan-Ranicki [DKR], and Davis-Quinn-Reich [DQR]) is that this Waldhausen Nil-group is isomorphic to the Bass Nil-group associated to the canonical index two subgroup $D_{4} \times \mathbb{Z}$ inside $D_{4} \times D_{\infty}$ (a considerable strengthening of the result of the authors in [LO08]). In our tables, we denote these groups by $\mathrm{Nil}_{0}=N K_{0}\left(\mathbb{Z} D_{4}\right)$ and $\mathrm{Nil}_{1}=N K_{1}\left(\mathbb{Z} D_{4}\right)$ (the lower Nil groups vanish by work of Farrell-Jones [FJ95]). These abelian groups are known to have the following properties:

(1) $\mathrm{Nil}_{1}$ is either trivial or infinitely generated [F77],

(2) $\mathrm{Nil}_{0}$ is infinitely generated (see below),

(3) in both of these groups, the order of every element divides 8 [G07, Corollary 5]. 
It is very likely that the group $\mathrm{Nil}_{1}$ is also non-trivial, but we were unable to establish this result. In order to see that $\mathrm{Nil}_{0}$ is non-trivial, consider the following Cartesian square:

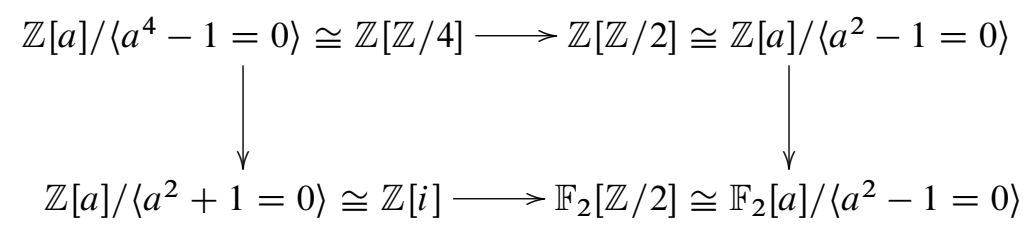

which yields the Cartesian square for $\mathbb{Z}\left[D_{4}\right]=\mathbb{Z}\left[\mathbb{Z} / 4 \rtimes_{\alpha} \mathbb{Z} / 2\right]=\mathbb{Z}[\mathbb{Z} / 4]_{\alpha}[\mathbb{Z} / 2]$,

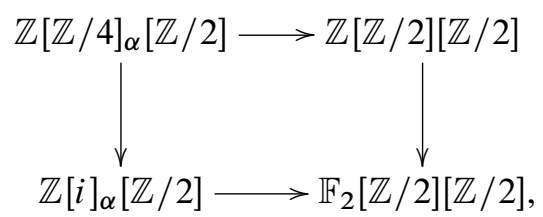

where in $\mathbb{Z}[i]_{\alpha}[\mathbb{Z} / 2]$, the automorphism $\alpha$ acts via $\alpha(i)=-i$. Writing $D_{2}=$ $\mathbb{Z} / 2 \times \mathbb{Z} / 2$ and $A=\mathbb{Z}[i]_{\alpha}[\mathbb{Z} / 2]$, and applying the $N K$-functor, this Cartesian square yields the Mayer-Vietoris sequence

$$
\begin{aligned}
N K_{2}\left(\mathbb{F}_{2}\left[D_{2}\right]\right) & \longrightarrow \mathrm{Nil}_{1} \longrightarrow N K_{1}\left(\mathbb{Z}\left[D_{2}\right]\right) \oplus N K_{1}(A) \longrightarrow N K_{1}\left(\mathbb{F}_{2}\left[D_{2}\right]\right) \\
& \longrightarrow \mathrm{Nil}_{0} \longrightarrow N K_{0}\left(\mathbb{Z}\left[D_{2}\right]\right) \oplus N K_{0}(A) \longrightarrow N K_{0}\left(\mathbb{F}_{2}\left[D_{2}\right]\right) .
\end{aligned}
$$

Several of the groups appearing in this Mayer-Vietoris sequence are known: the group $N K_{0}\left(\mathbb{F}_{2}\left[D_{2}\right]\right)$ vanishes by [Bas68], while the authors have previously shown [LO07] that the groups $N K_{1}\left(\mathbb{Z}\left[D_{2}\right]\right)$ and $N K_{0}\left(\mathbb{Z}\left[D_{2}\right]\right)$ are likewise countable infinite sums of $\mathbb{Z} / 2$.

Focusing on the tail end of the Mayer-Vietoris sequence, and substituting in the expressions we already know, we see that

$$
\cdots \longrightarrow \mathrm{Nil}_{0} \longrightarrow N K_{0}(A) \oplus \bigoplus_{\infty} \mathbb{Z} / 2 \longrightarrow 0,
$$

and non-triviality of $\mathrm{Nil}_{0}$ follows from the surjectivity onto the countable infinite sum of $\mathbb{Z} / 2$. In contrast, focusing on the head of the Mayer-Vietoris sequence, we see that

$$
N K_{2}\left(\mathbb{F}_{2}\left[D_{2}\right]\right) \longrightarrow \mathrm{Nil}_{1} \longrightarrow N K_{1}(A) \oplus \bigoplus_{\infty} \mathbb{Z} / 2 \longrightarrow N K_{1}\left(\mathbb{F}_{2}\left[D_{2}\right]\right) \longrightarrow \cdots
$$

Hence to establish that $\mathrm{Nil}_{1}$ is non-trivial from this sequence, one would need to either 
- establish that the first map is non-zero, i.e., understand the map

$$
N K_{2}\left(\mathbb{F}_{2}\left[D_{2}\right]\right) \rightarrow N K_{1}\left(\mathbb{Z} D_{4}\right)
$$

or

- establish that the second map is non-zero by showing that the third map has a non-trivial kernel, for instance by understanding the map $N K_{1}\left(\mathbb{Z}\left[D_{2}\right]\right) \rightarrow$ $N K_{1}\left(\mathbb{F}_{2}\left[D_{2}\right]\right)$.

The authors have some partial results concerning some of the terms showing up in the head of the Mayer-Vietoris sequence, but so far have been unsuccessful in establishing non-triviality of $\mathrm{Nil}_{1}$.

\section{The spectral sequences and final computations}

We now proceed to apply Corollary 3.4 to compute the lower algebraic $K$-theory of $\mathbb{Z} \Gamma$, for $\Gamma$ one of the 32 possible 3 -simplex hyperbolic reflection groups. Let us recall that Corollary 3.4 tells us that for such groups $\Gamma$ we have for $n \leq 1$ an isomorphism

$$
K_{n}(\mathbb{Z} \Gamma) \cong H_{n}^{\Gamma}\left(E_{\mathcal{V C}}(\Gamma) ; \mathbb{K} \mathbb{Z}^{-\infty}\right) \oplus \bigoplus_{i=1}^{k} H_{n}^{V_{i}}\left(E_{\mathcal{F} \mathcal{I N}}\left(V_{i}\right) \rightarrow *\right)
$$

where $\left\{V_{i}\right\}_{i=1}^{k}$ are a complete set of representatives for the conjugacy classes of maximal infinite virtually cyclic subgroups of type $\mathrm{I}$.

We first note that for all 32 of our groups, we have

- obtained in Section 4 a complete list of the type I maximal infinite virtually cyclic subgroups (listed out in Tables 2, 3, and 4),

- explicitly computed in Section 6 the groups

$$
H_{n}^{V}\left(E_{\mathscr{F} \mathcal{I N}}(V) \rightarrow *\right)
$$

for all the type I maximal infinite virtually cyclic subgroups that occur, with the exception of the case $V=D_{4} \times D_{\infty}$ (see Table 6).

In particular, this allows us to determine the expression

$$
\bigoplus_{i=1}^{k} H_{n}^{V_{i}}\left(E_{\mathcal{F} \mathcal{I N}}\left(V_{i}\right) \rightarrow *\right)
$$

occurring in the formula above for all 32 of our groups.

Hence we are left with computing $H_{n}^{\Gamma}\left(E_{\mathcal{F} \mathcal{I N}}(\Gamma) ; \mathbb{K} \mathbb{Z}^{-\infty}\right)$ for each of our 32 groups. In order to do this, we recall that Quinn [Qu82] established the existence of 
a spectral sequence which converges to this homology group, with $E^{2}$-terms given by

$$
E_{p, q}^{2}=H_{p}\left(E_{\mathcal{F} \mathcal{L N}}(\Gamma) / \Gamma ;\left\{\operatorname{Wh}_{q}\left(\Gamma_{\sigma}\right)\right\}\right) \Longrightarrow H_{n}^{\Gamma}\left(E_{\mathcal{F} \mathcal{L N}}(\Gamma) ; \mathbb{K} \mathbb{Z}^{-\infty}\right)
$$

The complex that gives the homology of $E_{\mathscr{F} \mathcal{L N}}(\Gamma) / \Gamma$ with local coefficients $\left\{\mathrm{Wh}_{q}\left(\Gamma_{\sigma}\right)\right\}$ has the form

$$
\begin{aligned}
\cdots \longrightarrow \bigoplus_{\sigma^{p+1}} \mathrm{Wh}_{q}\left(\Gamma_{\sigma^{p+1}}\right) \longrightarrow \bigoplus_{\sigma^{p}} \mathrm{Wh}_{q}\left(\Gamma_{\sigma^{p}}\right) \\
\quad \longrightarrow \bigoplus_{\sigma^{p-1}} \mathrm{Wh}_{q}\left(\Gamma_{\sigma^{p-1}}\right) \longrightarrow \cdots \longrightarrow \bigoplus_{\sigma^{0}} \mathrm{Wh}_{q}\left(\Gamma_{\sigma^{0}}\right),
\end{aligned}
$$

where $\sigma^{p}$ denotes the cells in dimension $p$, and the sum is over all $p$-dimensional cells in $E_{\mathscr{F} \mathcal{I N}}(\Gamma) / \Gamma$. The $p^{\text {th }}$ homology group of this complex will give us the entries for the $E_{p, q}^{2}$-term of the spectral sequence. Let us recall that

$$
\mathrm{Wh}_{q}(F)= \begin{cases}\mathrm{Wh}(F), & q=1, \\ \widetilde{K}_{0}(\mathbb{Z} F), & q=0, \\ K_{q}(\mathbb{Z} F), & q \leq-1 .\end{cases}
$$

Observe that for the groups we are interested in it is particularly easy to obtain a model for $E_{\mathcal{F} \mathcal{I N}}(\Gamma)$ : indeed, it is well known that for a lattice in $\operatorname{Isom}\left(\mathbb{H}^{n}\right)$ the action on $\mathbb{H}^{n}$ is a model for $E_{\mathcal{F} \mathcal{I N}}$. A cocompact model $\widehat{\mathbb{H}}^{3}$ can be obtained by equivariantly removing a suitable collection of horoballs from $\mathbb{H}^{3}$. In our specific situation, we obtain a model for $E_{\mathscr{F} I \mathcal{N}}(\Gamma)$ having a very explicit fundamental domain: the original hyperbolic 3-simplex, with the ideal vertices truncated.

Now note that for this fundamental domain $\widehat{\Delta}^{3}=\widehat{H}^{3} / \Gamma$, it is particularly easy to identify the stabilizers of each cell. Indeed, there will always be a single 3-dimensional cell, with trivial isotropy. There will be four 2-dimensional cells, each of which will have stabilizer $\mathbb{Z} / 2$. Additionally, there might be some 2-dimensional cells with trivial stabilizer (from the truncated vertices). Note that since $\mathrm{Wh}_{q}(1)$ and $\mathrm{Wh}_{q}(\mathbb{Z} / 2)$ vanish for all $q \leq 1$, this in particular implies that there will never be any contribution to the $E^{2}$-terms from the 3-dimensional and 2-dimensional cells. In other words, $E_{p, q}^{2}=0$ except possibly for $p=0,1$.

Now let us focus on the 1-dimensional and 0 -dimensional cells in the fundamental domain $\widehat{\Delta}^{3}$. We will have exactly six 1-dimensional cells, each of which will have stabilizer a dihedral group $D_{n}(n=2,3,4,5$, or 6$)$. In addition, there might be some 1-dimensional cells with stabilizer $\mathbb{Z} / 2$ (three such cells for each truncated vertex). Note that amongst these groups, the only ones that have some non-trivial $\mathrm{Wh}_{q}$ are the groups $D_{5}$ (for $q=1$ ) and $D_{6}$ (for $q=-1$ ). 
Next, we observe that there are at most four 0 -dimensional cells in $\widehat{\Delta}^{3}$ coming from the non-ideal vertices of $\Delta^{3}$. Furthermore, each of these vertices will have stabilizer a spherical Coxeter group, isomorphic to the special subgroup of the Coxeter group $\Gamma$ which corresponds to the vertex (up to isomorphism, these are the groups occurring in Table 1). The remaining vertices of $\widehat{\Delta}^{3}$ (coming from the truncated vertices in $\Delta^{3}$ ) will have stabilizers of the form $D_{n}(n=2,3,4,5$ or 6$)$. For all these groups, the non-vanishing $\mathrm{Wh}_{q}$ can be found in Table 5 .

Finally, we observe that since the only 1-cells with non-trivial $\mathrm{Wh}_{q}$ are the groups $D_{5}$ and $D_{6}$, most of the morphisms in the chain complex for the $E^{2}$-terms will either be zero (or in a few cases, will clearly be isomorphisms). The three morphisms one needs to take care with are the following:

- $K_{-1}\left(\mathbb{Z} D_{6}\right) \rightarrow K_{-1}\left(\mathbb{Z}\left[D_{6} \times \mathbb{Z} / 2\right]\right)$,

- $\mathrm{Wh}\left(D_{5}\right) \rightarrow \mathrm{Wh}\left(D_{10}\right)$,

- $\mathrm{Wh}\left(D_{5}\right) \rightarrow \mathrm{Wh}\left(A_{5} \times \mathbb{Z} / 2\right)$.

We proceed to analyze each of these three morphisms in the next three sections.

7.1. The map $K_{-1}\left(\mathbb{Z} D_{6}\right) \rightarrow K_{-1}\left(\mathbb{Z}\left[D_{6} \times \mathbb{Z} / 2\right]\right)$. We start by observing that $K_{-1}\left(\mathbb{Z} D_{6}\right) \cong \mathbb{Z}$ and $K_{-1}\left(\mathbb{Z}\left[D_{6} \times \mathbb{Z} / 2\right]\right) \cong \mathbb{Z}^{3}$ (see Table 5). We claim that the map induced by the natural inclusion $D_{6} \hookrightarrow D_{6} \times \mathbb{Z} / 2$ is injective, and the quotient group is isomorphic to $\mathbb{Z}^{2}$. In order to see this, we merely note that there is a retraction from $D_{6} \times \mathbb{Z} / 2$ to the subgroup $D_{6}$, and hence we must have that $K_{-1}\left(\mathbb{Z} D_{6}\right) \cong \mathbb{Z}$ is a summand inside $K_{-1}\left(\mathbb{Z}\left[D_{6} \times \mathbb{Z} / 2\right]\right) \cong \mathbb{Z}^{3}$, which immediately gives our claim.

7.2. The map $\mathbf{W h}\left(D_{5}\right) \rightarrow \operatorname{Wh}\left(D_{10}\right)$. We start by observing that $\operatorname{Wh}\left(D_{5}\right) \cong \mathbb{Z}$ and $\mathrm{Wh}\left(D_{10}\right) \cong \mathbb{Z}^{2}$ (see Table 5). We claim that the map induced by the natural inclusion $D_{5} \hookrightarrow D_{10} \cong D_{5} \times \mathbb{Z} / 2$ is injective, and the quotient group is isomorphic to $\mathbb{Z}$. But again, we see that there is a retraction from $D_{5} \times \mathbb{Z} / 2$ to the subgroup $D_{5}$, and hence $\mathrm{Wh}\left(D_{5}\right) \cong \mathbb{Z}$ is a summand inside $\operatorname{Wh}\left(D_{10}\right) \cong \mathbb{Z}^{2}$, which gives us our claim. Note that this map was used implicitly in Section 6.1 (in the argument mentioned in the second paragraph).

7.3. The map $\mathrm{Wh}\left(D_{5}\right) \rightarrow \mathrm{Wh}\left(A_{5} \times \mathbb{Z} / 2\right)$. We start by observing that $\mathrm{Wh}\left(D_{5}\right) \cong$ $\mathbb{Z}$ and $\mathrm{Wh}\left(A_{5} \times \mathbb{Z} / 2\right) \cong \mathbb{Z}^{2}$ (see Table 5). We claim that the map induced by the natural inclusion $D_{5} \hookrightarrow A_{5} \times \mathbb{Z} / 2$ is injective, and the quotient group is isomorphic to $\mathbb{Z}$. Note that in this case we do not have a retraction from the group $A_{5} \times \mathbb{Z} / 2$ to the subgroup $D_{5}$ (since $A_{5}$ is simple, the only possible non-trivial quotients would be isomorphic $\mathbb{Z} / 2, A_{5}$, or $A_{5} \times \mathbb{Z} / 2$ ).

Let us start by observing that, from the inclusion $D_{5} \hookrightarrow A_{5}$, we obtain that the inclusion $D_{5} \hookrightarrow A_{5} \times \mathbb{Z} / 2$ factors through

$$
D_{5} \hookrightarrow D_{5} \times \mathbb{Z} / 2 \cong D_{10} \hookrightarrow A_{5} \times \mathbb{Z} / 2
$$


which implies the map on Whitehead groups likewise factors through

$$
\mathrm{Wh}\left(D_{5}\right) \rightarrow \mathrm{Wh}\left(D_{10}\right) \rightarrow \mathrm{Wh}\left(A_{5} \times \mathbb{Z} / 2\right) .
$$

Observe that the first map in the above sequence was analyzed in the previous Section 7.2. Furthermore the last two groups in this sequence are abstractly isomorphic to $\mathbb{Z}^{2}$. So in order to obtain our claim, all we need to do is establish that the inclusion $D_{10} \hookrightarrow A_{5} \times \mathbb{Z} / 2$ induces an isomorphism on Whitehead groups.

In order to do this, we recall that Dress induction provides us with an isomorphism (see [O89, Chapter 11]):

$$
\mathrm{Wh}\left(A_{5} \times \mathbb{Z} / 2\right) \cong \underline{\lim }_{\longrightarrow} H \in \mathscr{H}\left(A_{5} \times \mathbb{Z} / 2\right) \mathrm{Wh}(H) .
$$

Here $\mathscr{H}\left(A_{5} \times \mathbb{Z} / 2\right)$ consists of all hyperelementary subgroups of $A_{5} \times \mathbb{Z} / 2$, the limit is over all maps induced by inclusion and conjugation, and the isomorphism is naturally induced by the inclusions. Now recall (Section 5.4) that the hyperelementary subgroups of $A_{5} \times \mathbb{Z} / 2$ are, up to isomorphism, $D_{2}, D_{2} \times \mathbb{Z} / 2, D_{3}, D_{5}, D_{6}$, and $D_{10}$. Amongst these groups (see Table 5), the only groups with non-trivial Wh are the groups $D_{5}$ and $D_{10}$, with $\mathrm{Wh}\left(D_{5}\right) \cong \mathbb{Z}$ and $\mathrm{Wh}\left(D_{10}\right) \cong \mathbb{Z}^{2}$. Furthermore, inside the group $A_{5} \times \mathbb{Z} / 2$, it is easy to see that

(1) every subgroup isomorphic to $D_{5}$ lies inside a subgroup isomorphic to $D_{10}$;

(2) all the subgroups isomorphic to $D_{10}$ are pairwise conjugate.

This immediately implies that the direct limit to the right is canonically isomorphic to $\mathrm{Wh}\left(D_{10}\right)$, which gives us our desired claim.

7.4. The spectral sequences. By this point of the paper we have

- described a simple model for $E_{\mathcal{F} \mathcal{I N}}(\Gamma)$ for our groups, and identified the stabilizers of cells (in this section),

- computed (in Section 5) the lower algebraic $K$-groups of the stabilizers of the cells, and

- identified (in this section) the non-trivial morphisms appearing in the computation of the $E^{2}$-terms of the Quinn spectral sequence.

Furthermore, as explained earlier, the only possible non-zero terms in the spectral sequence are the $E_{p, q}$ with $p=0,1$. This boils down to understanding the homology of the complex

$$
0 \longrightarrow \bigoplus_{\sigma^{1}} \mathrm{Wh}_{q}\left(\Gamma_{\sigma^{1}}\right) \longrightarrow \bigoplus_{\sigma^{0}} \mathrm{Wh}_{q}\left(\Gamma_{\sigma^{0}}\right) \longrightarrow 0 .
$$

But we have seen (Sections 7.1, 7.2, and 7.3) that the middle map is always injective, hence the $E_{1, q}$ terms will also vanish. This gives us that in all 32 cases the spectral 
sequence collapses at the $E^{2}$-term. In fact, in all 32 cases, the only possible non-zero $E^{2}$-terms are $E_{0,-1}^{2}, E_{0,0}^{2}, E_{0,1}^{2}$. In particular the $K_{i}(\mathbb{Z} \Gamma)$ vanish for $i \leq-2$.

The results obtained for $K_{-1}, \widetilde{K}_{0}$, and Wh for all 32 hyperbolic 3-simplex groups are listed out in Table 7 and Table 8 (on the next two pages). For ease of notation, we have only entered the non-zero terms in the tables; all the blank squares represent entries where the corresponding group vanishes.

Table 7. Lower algebraic $K$-theory of non-cocompact hyperbolic 3-simplex groups.

\begin{tabular}{|c|c|c|c|}
\hline$\Gamma$ & $K_{-1} \neq 0$ & $\tilde{K}_{0} \neq 0$ & $\mathrm{Wh} \neq 0$ \\
\hline \multicolumn{4}{|l|}{$\left[3^{[] \times[]}\right]$} \\
\hline \multicolumn{4}{|l|}{$\left[4^{[4]}\right]$} \\
\hline$\left[(3,6)^{[2]}\right]$ & $\mathbb{Z}^{2}$ & & \\
\hline$\left[6,3^{[3]}\right]$ & $\mathbb{Z}^{2}$ & & \\
\hline$\left[4^{1,1,1}\right]$ & & $\mathbb{Z} / 2$ & \\
\hline$[(3,5,3,6)]$ & $\mathbb{Z}^{5}$ & $(\mathbb{Z} / 2)^{2}$ & $\mathbb{Z}^{3}$ \\
\hline$\left[\left(3,4^{3}\right)\right]$ & $\mathbb{Z}^{2}$ & $(\mathbb{Z} / 4)^{2}$ & \\
\hline$[(3,4,3,6)]$ & $\mathbb{Z}^{3}$ & $(\mathbb{Z} / 4)^{2} \oplus \mathrm{Nil}_{0}$ & $\mathrm{Nil}_{1}$ \\
\hline$\left[\left(3^{3}, 6\right)\right]$ & $\mathbb{Z}$ & & \\
\hline$\left[3^{[3,3]}\right]$ & & $\bigoplus_{\infty} \mathbb{Z} / 2$ & $\bigoplus_{\infty} \mathbb{Z} / 2$ \\
\hline$\left[6,3^{1,1}\right]$ & $\mathbb{Z}$ & $\bigoplus_{\infty} \mathbb{Z} / 2$ & $\bigoplus_{\infty} \mathbb{Z} / 2$ \\
\hline$[3,6,3]$ & $\mathbb{Z}^{3}$ & & \\
\hline$[6,3,6]$ & $\mathbb{Z}^{6}$ & $(\mathbb{Z} / 2)^{4}$ & \\
\hline$[4,4,4]$ & & $(\mathbb{Z} / 4)^{2} \oplus \bigoplus_{\infty} \mathbb{Z} / 2$ & $\bigoplus_{\infty} \mathbb{Z} / 2$ \\
\hline$\left[5,3^{[3]}\right]$ & $\mathbb{Z}^{4}$ & $\bigoplus_{\infty} \mathbb{Z} / 2$ & $\mathbb{Z}^{3} \oplus \bigoplus_{\infty} \mathbb{Z} / 2$ \\
\hline$[5,3,6]$ & $\mathbb{Z}^{6}$ & $\bigoplus_{\infty} \mathbb{Z} / 2$ & $\mathbb{Z}^{3} \oplus \bigoplus_{\infty} \mathbb{Z} / 2$ \\
\hline$\left[\left(3^{2}, 4^{2}\right)\right]$ & $\mathbb{Z}^{2}$ & $(\mathbb{Z} / 4)^{2} \oplus \bigoplus_{\infty} \mathbb{Z} / 2$ & $\bigoplus_{\infty} \mathbb{Z} / 2$ \\
\hline$\left[4,3^{[3]}\right]$ & $\mathbb{Z}^{3}$ & $(\mathbb{Z} / 4)^{2} \oplus \bigoplus_{\infty} \mathbb{Z} / 2 \oplus \mathrm{Nil}_{0}$ & $\bigoplus_{\infty} \mathbb{Z} / 2 \oplus \mathrm{Nil}_{1}$ \\
\hline$\left[3,3^{[3]}\right]$ & & $\bigoplus_{\infty} \mathbb{Z} / 2$ & $\bigoplus_{\infty} \mathbb{Z} / 2$ \\
\hline$\left[3,4^{1,1}\right]$ & $\mathbb{Z}^{2}$ & $(\mathbb{Z} / 4)^{2} \oplus \bigoplus_{\infty} \mathbb{Z} / 2$ & $\bigoplus_{\infty} \mathbb{Z} / 2$ \\
\hline$[4,3,6]$ & $\mathbb{Z}^{4}$ & $(\mathbb{Z} / 4)^{2} \oplus \bigoplus_{\infty} \mathbb{Z} / 2 \oplus \mathrm{Nil}_{0}$ & $\bigoplus_{\infty} \mathbb{Z} / 2 \oplus \mathrm{Nil}_{1}$ \\
\hline$[3,3,6]$ & $\mathbb{Z}^{4}$ & $\bigoplus_{\infty} \mathbb{Z} / 2$ & $\bigoplus_{\infty} \mathbb{Z} / 2$ \\
\hline$[3,4,4]$ & $\mathbb{Z}^{2}$ & $(\mathbb{Z} / 4)^{2} \oplus \bigoplus_{\infty} \mathbb{Z} / 2$ & $\bigoplus_{\infty} \mathbb{Z} / 2$ \\
\hline
\end{tabular}


Table 8. Lower algebraic $K$-theory of cocompact hyperbolic 3 -simplex groups.

\begin{tabular}{|c|c|c|c|}
\hline$\Gamma$ & $K_{-1} \neq 0$ & $\tilde{K}_{0} \neq 0$ & $\mathrm{Wh} \neq 0$ \\
\hline$[3,5,3]$ & $\mathbb{Z}^{6}$ & $\bigoplus_{\infty} \mathbb{Z} / 2$ & $\mathbb{Z}^{3} \oplus \oplus_{\infty} \mathbb{Z} / 2$ \\
\hline$[5,3,5]$ & $\mathbb{Z}^{6}$ & $\bigoplus_{\infty} \mathbb{Z} / 2$ & $\mathbb{Z}^{6} \oplus \oplus_{\infty} \mathbb{Z} / 2$ \\
\hline$\left[\left(3^{3}, 4\right)\right]$ & $\mathbb{Z}^{2}$ & $(\mathbb{Z} / 4)^{2} \oplus \bigoplus_{\infty} \mathbb{Z} / 2 \oplus \mathrm{Nil}_{0}$ & $\bigoplus_{\infty} \mathbb{Z} / 2 \oplus \mathrm{Nil}_{1}$ \\
\hline$\left[5,3^{1,1}\right]$ & $\mathbb{Z}^{4}$ & $\bigoplus_{\infty} \mathbb{Z} / 2$ & $\mathbb{Z}^{3} \oplus \oplus_{\infty} \mathbb{Z} / 2$ \\
\hline$[4,3,5]$ & $\mathbb{Z}^{4}$ & $(\mathbb{Z} / 4)^{2} \oplus \bigoplus_{\infty} \mathbb{Z} / 2 \oplus \mathrm{Nil}_{0}$ & $\mathbb{Z}^{3} \oplus \bigoplus_{\infty} \mathbb{Z} / 2 \oplus \mathrm{Nil}_{1}$ \\
\hline$\left[\left(3^{3}, 5\right)\right]$ & $\mathbb{Z}^{4}$ & $\bigoplus_{\infty} \mathbb{Z} / 2$ & $\mathbb{Z}^{3} \oplus \bigoplus_{\infty} \mathbb{Z} / 2$ \\
\hline$\left[(3,5)^{[2]}\right]$ & $\mathbb{Z}^{8}$ & $\bigoplus_{\infty} \mathbb{Z} / 2$ & $\mathbb{Z}^{6} \oplus \oplus_{\infty} \mathbb{Z} / 2$ \\
\hline$\left[(3,4)^{[2]}\right]$ & $\mathbb{Z}^{4}$ & $(\mathbb{Z} / 4)^{4} \oplus \bigoplus_{\infty} \mathbb{Z} / 2 \oplus\left(2 \cdot \mathrm{Nil}_{0}\right)$ & $\oplus_{\infty} \mathbb{Z} / 2 \oplus\left(2 \cdot \mathrm{Nil}_{1}\right)$ \\
\hline$[(3,4,3,5)]$ & $\mathbb{Z}^{6}$ & $(\mathbb{Z} / 4)^{2} \oplus \bigoplus_{\infty} \mathbb{Z} / 2 \oplus \mathrm{Nil}_{0}$ & $\mathbb{Z}^{3} \oplus \oplus_{\infty} \mathbb{Z} / 2 \oplus \mathrm{Nil}_{1}$ \\
\hline
\end{tabular}

Note that several of the group appearing in Tables 7 and 8 involve copies of the Bass Nil-groups $N K_{0}\left(\mathbb{Z} D_{4}\right)$ and $N K_{1}\left(\mathbb{Z} D_{4}\right)$ (see Section 5.5). In order to simplify the notation in the tables, we used $\mathrm{Nil}_{0}$ and $\mathrm{Nil}_{1}$ to denote these two Nil-groups. Recall that we know that the groups $\mathrm{Nil}_{0}, \mathrm{Nil}_{1}$ are torsion groups, where the order of every element divides 8 , and furthermore the group $\mathrm{Nil}_{0}$ is infinitely generated (see Section 5.5). In the entries for the group $\left[(3,4)^{[2]}\right]$, the expression $2 \cdot \mathrm{Nil}_{i}$ denotes two copies of the Bass Nil-group $\mathrm{Nil}_{i}$.

Remark. In an Addendum to the present paper [We], C. Weibel has obtained some additional information on the Bass Nil-groups $\mathrm{Nil}_{0}, \mathrm{Nil}_{1}$. Specifically, he shows that $\mathrm{Nil}_{0}$ is the direct sum of a countably infinite free $\mathbb{Z} / 2$-module with a countably infinite free $\mathbb{Z} / 4$-module. He also shows that $\mathrm{Nil}_{1}$ is a countably infinite torsion group of exponent 2 or 4 .

\section{Appendix: two specific examples}

In this Appendix we work through the entire procedure for two specific examples (one cocompact, and one non-cocompact), with a view of helping the reader understand the layout of the paper. 
8.1. The group $\left[(3,5)^{[2]}\right]$. The Coxeter diagram for this group $\Gamma$ can be found in Figure 1, from which the following presentation can be read off (see Section 2):

$$
\begin{gathered}
\langle w, x, y, z| w^{2}=x^{2}=y^{2}=z^{2}=1, \\
\left.(w x)^{3}=(x y)^{5}=(y z)^{3}=(z w)^{5}=(w y)^{2}=(x z)^{2}=1\right\rangle .
\end{gathered}
$$

This group acts on $\mathbb{H}^{3}$ cocompactly, with fundamental domain a 3 -simplex $\Delta^{3}$. After labeling the hyperplanes extending the four faces by the four generators of $\Gamma$, the angles between these hyperplanes satisfy the following relationships (see Section 2):

- $\angle\left(P_{w}, P_{y}\right)=\angle\left(P_{x}, P_{z}\right)=\pi / 2$,

- $\angle\left(P_{w}, P_{x}\right)=\angle\left(P_{y}, P_{z}\right)=\pi / 3$,

- $\angle\left(P_{w}, P_{z}\right)=\angle\left(P_{x}, P_{y}\right)=\pi / 5$.

In particular, the action of $\Gamma$ on $\mathbb{H}^{3}$ gives a cocompact model for $E_{\mathscr{F} I \mathcal{N}}(\Gamma)$, and the splitting formula (see Corollary 3.4) tells us that we have, for all $n \leq 1$, isomorphisms

$$
K_{n}(\mathbb{Z} \Gamma) \cong H_{n}^{\Gamma}\left(E_{\mathscr{F} \mathcal{I N}}(\Gamma) ; \mathbb{K} \mathbb{Z}^{-\infty}\right) \oplus \bigoplus_{i=1}^{k} H_{n}^{V_{i}}\left(E_{\mathcal{F} \mathcal{I N}}\left(V_{i}\right) \rightarrow *\right)
$$

Let us now identify the (finitely many) groups $\left\{V_{i}\right\}$ that appear in the above formula. As explained in Section 4, these groups will arise as stabilizers of type I geodesics, which are precisely (up to the $\Gamma$-action) one of the six geodesics $P_{w} \cap P_{x}$, $P_{w} \cap P_{y}, P_{w} \cap P_{z}, P_{x} \cap P_{y}, P_{x} \cap P_{z}$, and $P_{y} \cap P_{z}$. To identify the stabilizers of these geodesics, we first need to identify the vertex stabilizers for the simplex $\Delta^{3}$. Recall that these will be the special subgroups generated by triples of generators. But from the Coxeter diagram for $\Gamma$, one immediately sees that any triple of vertices spans out a subdiagram corresponding to the Coxeter group $[3,5]$. This implies that every vertex has stabilizer isomorphic to the (finite) Coxeter group [3,5], which is well known to be isomorphic to the group $A_{5} \times \mathbb{Z} / 2$. Now for each of the six type I geodesics we have, one can consider the projection to the fundamental domain $\Delta^{3}$. From Table 1, looking up the vertex stabilizers $A_{5} \times \mathbb{Z} / 2$, we see that every one of the six geodesics projects to precisely the associated edge in $\Delta^{3}$. Now to find the stabilizers of the geodesics, one applies Bass-Serre theory. The stabilizer acts on each of the geodesics with quotient a segment, so one can write each of the stabilizers as a generalized free product. Furthermore, Table 1 allows us to identify the vertex groups in the Bass-Serre graph of groups.

Let us see how this works, for instance in the case of the geodesic $P_{x} \cap P_{y}$. The two associated hyperplanes $P_{x}$ and $P_{y}$ intersect at an angle of $\pi / 5$, hence the edge group in the Bass-Serre graph of groups will be $D_{5}$. For the vertex groups, we see that the corresponding segment in $\Delta^{3}$ joins a pair of vertices with stabilizer 
$A_{5} \times \mathbb{Z} / 2$, and correspond to the angle of $\pi / 5$ at both the vertices. The last row in Table 1 tells us that both the vertex groups in the Bass-Serre graph of groups will be $D_{10}$. This tells us that the stabilizer of the geodesic $P_{x} \cap P_{y}$ is precisely the group $D_{10} * D_{5} D_{10} \cong D_{5} \times D_{\infty}$. Carrying this procedure out for each of the six geodesics, one finds that the stabilizers one obtains are as follows:

- two copies of $D_{10} *_{D_{5}} D_{10}$, corresponding to the two geodesics $P_{x} \cap P_{y}$ and $P_{w} \cap P_{z}$,

- two copies of $D_{6} *_{D_{3}} D_{6}$, corresponding to the two geodesics $P_{w} \cap P_{x}$ and $P_{y} \cap P_{z}$,

- two copies of $D_{2} \times D_{\infty}$, corresponding to the two geodesics $P_{w} \cap P_{y}$ and $P_{x} \cap P_{z}$.

Note that these are precisely the groups that are listed out in Table 4. Finally, amongst these six subgroups, one needs to know which ones have a non-trivial cokernel for the relative assembly map. But from the work in Section 6, all the non-trivial cokernels are listed out in Table 6. Looking up Table 6, one sees that out of these six groups, the only ones with non-trivial cokernels are the two copies of $D_{2} \times D_{\infty}$, each of whom contributes $\bigoplus_{\infty} \mathbb{Z} / 2$ to the $K_{0}(\mathbb{Z} \Gamma)$ and $\mathrm{Wh}(\Gamma)$.

So we are finally left with computing the homology coming from the finite subgroups, i.e., the term $H_{n}^{\Gamma}\left(E_{\mathcal{F} \mathcal{I N}}(\Gamma) ; \mathbb{K}^{-\infty}\right)$. As we mentioned earlier, a cocompact fundamental domain for $\mathbb{H}^{3} / \Gamma$ is given by $\Delta^{3}$. The stabilizers of cells in the fundamental domain can be read off from the Coxeter diagram, as they will precisely be the special subgroups (see the discussion in Section 7). We see that

- there is one 3-dimensional cell (the interior of $\Delta^{3}$ ), with trivial stabilizer,

- there are four 2-dimensional cells (the faces of $\Delta^{3}$ ), with stabilizer $\mathbb{Z} / 2$,

- there are six 1-dimensional cells (the edges of $\Delta^{3}$ ), two of which have stabilizer $D_{2}$, two of which have stabilizer $D_{3}$, and two of which have stabilizer $D_{5}$,

- there are four 0-dimensional cells (the vertices of $\Delta^{3}$ ), each of which has stabilizer $A_{5} \times \mathbb{Z} / 2$.

Now to obtain the $E^{2}$-terms in the Quinn spectral sequence, we need the homology of the complex

$$
\begin{aligned}
\cdots \longrightarrow & \bigoplus_{\sigma^{p+1}} \mathrm{Wh}_{q}\left(\Gamma_{\sigma^{p+1}}\right) \longrightarrow \bigoplus_{\sigma^{p}} \mathrm{Wh}_{q}\left(\Gamma_{\sigma^{p}}\right) \\
& \longrightarrow \bigoplus_{\sigma^{p-1}} \mathrm{Wh}_{q}\left(\Gamma_{\sigma^{p-1}}\right) \longrightarrow \cdots \longrightarrow \bigoplus_{\sigma^{0}} \mathrm{Wh}_{q}\left(\Gamma_{\sigma^{0}}\right),
\end{aligned}
$$

where $\sigma^{p}$ are the $p$-dimensional cells (which we identified above). But from the work in Section 5, we know explicitly all the groups appearing in the above complex. 
Indeed, looking up the non-zero $K$-groups in Table 5, we see that for $q<-1$, the entire complex is identically zero. We now consider the remaining values $q=-1,0,1$.

$q=-1:$ The complex degenerates to

$$
0 \rightarrow 4 K_{-1}\left(\mathbb{Z}\left[A_{5} \times \mathbb{Z} / 2\right]\right) \rightarrow 0,
$$

where the four copies of $K_{-1}\left(\mathbb{Z}\left[A_{5} \times \mathbb{Z} / 2\right]\right)$ come from the four vertices of $\Delta^{3}$. Since we know (see Table 5) that $K_{-1}\left(\mathbb{Z}\left[A_{5} \times \mathbb{Z} / 2\right]\right) \cong \mathbb{Z}^{2}$, we immediately get that $E_{p,-1}^{2}$ all vanish, with the exception of $E_{0,-1}^{2} \cong \mathbb{Z}^{8}$.

$q=0$ : The complex degenerates to

$$
0 \rightarrow 4 \widetilde{K}_{0}\left(\mathbb{Z}\left[A_{5} \times \mathbb{Z} / 2\right]\right) \rightarrow 0,
$$

where the four copies of $\widetilde{K}_{0}\left(\mathbb{Z}\left[A_{5} \times \mathbb{Z} / 2\right]\right)$ come from the four vertices of $\Delta^{3}$. Since we know (see Table 5) that $\widetilde{K}_{0}\left(\mathbb{Z}\left[A_{5} \times \mathbb{Z} / 2\right]\right) \cong \mathbb{Z} / 2$, we immediately get that $E_{p, 0}^{2}$ all vanish, with the exception of $E_{0,0}^{2} \cong(\mathbb{Z} / 2)^{4}$.

$q=1:$ The complex degenerates to

$$
0 \rightarrow 2 \mathrm{Wh}\left(D_{5}\right) \rightarrow 4 \mathrm{Wh}\left(A_{5} \times \mathbb{Z} / 2\right) \rightarrow 0
$$

Note that the first copy of $\operatorname{Wh}\left(D_{5}\right)$ comes from the edge $P_{x} \cap P_{y} \cap \Delta^{3}$, while the second copy of $\mathrm{Wh}\left(D_{5}\right)$ comes from the edge $P_{w} \cap P_{z} \cap \Delta^{3}$. The four copies of $\mathrm{Wh}\left(A_{5} \times \mathbb{Z} / 2\right)$ come from the four vertices of $\Delta^{3}$.

Since the two edges $P_{x} \cap P_{y} \cap \Delta^{3}$ and $P_{w} \cap P_{z} \cap \Delta^{3}$ are disjoint, the complex splits as a sum of two subcomplexes, one for each of the two edges. Focusing on the first edge, we see that we have

$$
0 \rightarrow \mathrm{Wh}\left(D_{5}\right) \rightarrow 2 \mathrm{Wh}\left(A_{5} \times \mathbb{Z} / 2\right) \rightarrow 0 .
$$

We know that $\mathrm{Wh}\left(D_{5}\right) \cong \mathbb{Z}$ and $\mathrm{Wh}\left(A_{5} \times \mathbb{Z} / 2\right) \cong \mathbb{Z}^{2}$ (see Table 5), and that the map $\mathrm{Wh}\left(D_{5}\right) \hookrightarrow \mathrm{Wh}\left(A_{5} \times \mathbb{Z} / 2\right)$ induced by inclusion is split injective (see Section 7.3). This immediately tells us that in the chain complex above, we have that $2 \mathrm{Wh}\left(A_{5} \times \mathbb{Z} / 2\right) / \mathrm{Wh}\left(D_{5}\right) \cong \mathbb{Z}^{3}$. An identical analysis for the other edge gives us that the homology of the original complex yields $E_{1,1}^{2} \cong 0$ and $E_{0,1}^{2} \cong \mathbb{Z}^{6}$.

Combining everything we have said so far, we see that for the Quinn spectral sequence, the only non-zero $E^{2}$-terms are $E_{0,-1}^{2} \cong \mathbb{Z}^{8}$ and $E_{0,1}^{2} \cong \mathbb{Z}^{6}$. This implies that the spectral sequence immediately collapses, giving us that

$$
H_{n}^{\Gamma}\left(E_{\mathscr{F} \mathcal{I N}}(\Gamma) ; \mathbb{K}^{-\infty}\right) \cong 0, \quad \text { for } n<-1,
$$

and

$$
\begin{aligned}
& H_{-1}^{\Gamma}\left(E_{\mathcal{F} \mathcal{L N}}(\Gamma) ; \mathbb{K}^{-\infty}\right) \cong \mathbb{Z}^{8}, \\
& H_{0}^{\Gamma}\left(E_{\mathcal{F} \mathcal{I N}}(\Gamma) ; \mathbb{K} \mathbb{Z}^{-\infty}\right) \cong(\mathbb{Z} / 2)^{4}, \\
& H_{1}^{\Gamma}\left(E_{\mathcal{F} \mathcal{I N}}(\Gamma) ; \mathbb{K} \mathbb{Z}^{-\infty}\right) \cong \mathbb{Z}^{6} .
\end{aligned}
$$


We now have both the terms appearing in the splitting formula, and we conclude that the lower algebraic $K$-theory of the group $\Gamma$ is given by

$$
\mathrm{Wh}_{n}(\Gamma)= \begin{cases}\mathrm{Wh}(\Gamma) \cong \mathbb{Z}^{6} \oplus \bigoplus_{\infty} \mathbb{Z} / 2, & n=1 \\ \widetilde{K}_{0}(\mathbb{Z} \Gamma) \cong \bigoplus_{\infty} \mathbb{Z} / 2, & n=0 \\ K_{-1}(\mathbb{Z} \Gamma) \cong \mathbb{Z}^{8}, & n=-1 \\ K_{n}(\mathbb{Z} \Gamma) \cong 0, & n \leq-1\end{cases}
$$

Looking up Table 8, one finds that these are precisely the values reported.

8.2. The group $\left[3,4^{1,1}\right]$. The Coxeter diagram for this group $\Gamma$ can be found in Figure 2, from which the following presentation can be read off (see Section 2):

$$
\begin{gathered}
\langle w, x, y, z| w^{2}=x^{2}=y^{2}=z^{2}=1, \\
\left.(w x)^{3}=(x y)^{4}=(y z)^{2}=(z w)^{2}=(w y)^{2}=(x z)^{4}=1\right\rangle .
\end{gathered}
$$

This group acts on $\mathbb{H}^{3}$ with cofinite volume, with fundamental domain a (noncompact) 3-simplex $\Delta^{3}$ with one ideal vertex. After labeling the hyperplanes extending the four faces by the four generators of $\Gamma$, the angles between these hyperplanes satisfy the following relationships (see Section 2):

- $\angle\left(P_{w}, P_{y}\right)=\angle\left(P_{w}, P_{z}\right)=\angle\left(P_{w}, P_{z}\right)=\pi / 2$,

- $\angle\left(P_{w}, P_{x}\right)=\pi / 3$,

- $\angle\left(P_{x}, P_{z}\right)=\angle\left(P_{x}, P_{y}\right)=\pi / 4$.

The ideal vertex arises as the intersection (at infinity) of the three hyperplanes $P_{x} \cap$ $P_{y} \cap P_{z}$, and has stabilizer the 2-dimensional crystallographic group [4, 4].

Now as discussed in Section 7, the action of $\Gamma$ on $\mathbb{H}^{3}$ provides us with a model for $E_{\mathcal{F} \mathcal{I N}}(\Gamma)$. Furthermore, the splitting formula (see Corollary 3.4) tells us that we have, for all $n \leq 1$, isomorphisms

$$
K_{n}(\mathbb{Z} \Gamma) \cong H_{n}^{\Gamma}\left(E_{\mathcal{F} \mathcal{I N}}(\Gamma) ; \mathbb{K} \mathbb{Z}^{-\infty}\right) \oplus \bigoplus_{i=1}^{k} H_{n}^{V_{i}}\left(E_{\mathcal{F} \mathcal{I N}}\left(V_{i}\right) \rightarrow *\right)
$$

Let us now identify the (finitely many) groups $\left\{V_{i}\right\}$ that appear in the above formula. As explained in Section 4, these groups will arise as stabilizers of type I geodesics, which are precisely (up to the $\Gamma$-action) one of the six geodesics $P_{w} \cap P_{x}$, $P_{w} \cap P_{y}, P_{w} \cap P_{z}, P_{x} \cap P_{y}, P_{x} \cap P_{z}$, and $P_{y} \cap P_{z}$. Note that since the geodesic segments $P_{x} \cap P_{y}, P_{y} \cap P_{z}$ and $P_{x} \cap P_{z}$ project to non-compact segments in the fundamental domain (they give rise to edges joined to the ideal vertex), these geodesics will never have an infinite stabilizer, and we can hence safely ignore them. 
To identify the stabilizers of the remaining three geodesics, we follow the procedure from Section 4. We first need to identify the (non-ideal) vertex stabilizers for the simplex $\Delta^{3}$. Recall that these will be the special subgroups generated by triples of generators. But from the Coxeter diagram for $\Gamma$, and the subdiagram spanned out by the triple of vertices, we obtain that:

- the Coxeter group $[3,4] \cong S_{4} \times \mathbb{Z} / 2$ will be the stabilizer of the vertices $P_{w} \cap$ $P_{x} \cap P_{z}$ and of the vertex $P_{w} \cap P_{x} \cap P_{y}$,

- the group $(\mathbb{Z} / 2)^{3}$ will be the stabilizer of the vertex $P_{w} \cap P_{y} \cap P_{z}$.

Now for each of the three (potentially cocompact) type I geodesics that we have ( $P_{w} \cap P_{x}, P_{w} \cap P_{y}$, and $P_{w} \cap P_{z}$ ) one can consider the projection to the fundamental domain $\Delta^{3}$. From Table 1, looking up the vertex stabilizers $S_{4} \times \mathbb{Z} / 2$, we see that every one of the three geodesics projects to precisely the associated edge in $\Delta^{3}$.

To find the stabilizers of these geodesics, we now use Bass-Serre theory as explained in Section 4. To find the vertex groups, one uses Table 1, while the edge group will be precisely the dihedral group given by the special subgroup associated to the geodesic. This immediately gives us the stabilizers:

- one copy of $D_{6} *_{D_{3}} D_{6}$, corresponding to the geodesic $P_{w} \cap P_{x}$,

- two copies of $\left(\mathbb{Z} / 2 \times D_{2}\right) * D_{2}\left(\mathbb{Z} / 2 \times D_{2}\right) \cong D_{2} \times D_{\infty}$, corresponding to the two geodesics $P_{w} \cap P_{y}$ and $P_{w} \cap P_{z}$,

which are precisely the groups reported in Table 3. Finally, amongst these three subgroups, one needs to decide which ones have a non-trivial cokernel for the relative assembly map. These cokernels are listed out in Table 6 , and one sees that the only non-trivial contribution will come from the two copies of $D_{2} \times D_{\infty}$, each of which will contribute $\bigoplus_{\infty} \mathbb{Z} / 2$ to the $\widetilde{K}_{0}(\mathbb{Z} \Gamma)$ and $\mathrm{Wh}(\Gamma)$.

So we are finally left with computing the homology coming from the finite subgroups, i.e., the term $H_{n}^{\Gamma}\left(E_{\mathcal{F} \mathcal{I N}}(\Gamma) ; \mathbb{K}^{-\infty}\right)$. As we mentioned earlier, a fundamental domain for $\widehat{\mathbb{H}}^{3} / \Gamma$ is given by the original hyperbolic 3-simplex $\Delta^{3}$ with the ideal vertex $P_{x} \cap P_{y} \cap P_{z}$ truncated. The stabilizers of cells in the fundamental domain can be read off from the Coxeter diagram, as they will precisely be the special subgroups (see the discussion in Section 7). We see that

- there is one 3-dimensional cell (the interior of $\Delta^{3}$ ), with trivial stabilizer;

- there are four 2-dimensional cells with stabilizer $\mathbb{Z} / 2$ (for the faces of the original $\Delta^{3}$ ), and one 2-dimensional cell with trivial stabilizer (from the truncated vertex);

- there are nine 1-dimensional cells: three of these have stabilizer $D_{2}$ (from the edges corresponding to $P_{y} \cap P_{z}, P_{w} \cap P_{y}$, and $P_{w} \cap P_{z}$ ), two have stabilizer $D_{4}$ (from the edges corresponding to $P_{x} \cap P_{y}$ and $P_{x} \cap P_{z}$ ), one has stabilizer $D_{3}$ (from the edge corresponding to $P_{w} \cap P_{x}$ ), and three have stabilizer $\mathbb{Z} / 2$ (from the truncation of $P_{x}, P_{y}, P_{z}$ ); 
- there are six 0-dimensional cells: two have stabilizer $S_{4} \times \mathbb{Z} / 2$ (from two of the non-ideal vertices), one has stabilizer $D_{2} \times \mathbb{Z} / 2$ (from the third non-ideal vertex), two have stabilizer $D_{4}$ (from the truncation of the edges $P_{x} \cap P_{y}$ and $P_{x} \cap P_{z}$ ), and one has stabilizer $D_{2}$ (from the truncation of the edge $P_{y} \cap P_{z}$ ).

Now to obtain the $E^{2}$-terms in the Quinn spectral sequence, we need the homology of the complex

$$
\begin{aligned}
\cdots \longrightarrow & \bigoplus_{\sigma^{p+1}} \mathrm{Wh}_{q}\left(\Gamma_{\sigma^{p+1}}\right) \longrightarrow \bigoplus_{\sigma^{p}} \mathrm{Wh}_{q}\left(\Gamma_{\sigma^{p}}\right) \\
& \longrightarrow \bigoplus_{\sigma^{p-1}} \mathrm{Wh}_{q}\left(\Gamma_{\sigma^{p-1}}\right) \longrightarrow \cdots \longrightarrow \bigoplus_{\sigma^{0}} \mathrm{Wh}_{q}\left(\Gamma_{\sigma^{0}}\right),
\end{aligned}
$$

where $\sigma^{p}$ are the $p$-dimensional cells (which we identified above). But from the work in Section 5, we know explicitly all the groups appearing in the above complex. Indeed, looking up the non-zero $K$-groups in Table 5, we see that the only two of the cell stabilizers that have non-trivial $K$-theory are the groups $D_{2} \times \mathbb{Z} / 2$ and $S_{4} \times \mathbb{Z} / 2$. There are two copies of the group $S_{4} \times \mathbb{Z} / 2$, arising as stabilizers of 0 -cells, and we have that $K_{-1}\left(\mathbb{Z}\left[S_{4} \times \mathbb{Z} / 2\right]\right) \cong \mathbb{Z}$ and $\widetilde{K}_{0}\left(\mathbb{Z}\left[S_{4} \times \mathbb{Z} / 2\right]\right) \cong \mathbb{Z} / 4$. There is only one copy of the group $D_{2} \times \mathbb{Z} / 2$, arising as the stabilizer of one single 0 -cell, and we have that $\widetilde{K}_{0}\left(\mathbb{Z}\left[D_{2} \times \mathbb{Z} / 2\right]\right) \cong \mathbb{Z} / 2$. This immediately tells us that non-zero terms in the Quinn spectral sequence will be $E_{0,-1}^{2} \cong \mathbb{Z}^{2}$ and $E_{0,0}^{2} \cong(\mathbb{Z} / 4)^{2} \oplus \mathbb{Z} / 2$. This implies that the spectral sequence immediately collapses, giving us that

$$
H_{n}^{\Gamma}\left(E_{\mathcal{F} \mathcal{I N}}(\Gamma) ; \mathbb{K}^{-\infty}\right) \cong 0
$$

for $n<-1, n=1$, and

$$
\begin{gathered}
H_{-1}^{\Gamma}\left(E_{\mathcal{F} \mathcal{I N}}(\Gamma) ; \mathbb{K} \mathbb{Z}^{-\infty}\right) \cong \mathbb{Z}^{2}, \\
H_{0}^{\Gamma}\left(E_{\mathcal{F} \mathcal{I N}}(\Gamma) ; \mathbb{K} \mathbb{Z}^{-\infty}\right) \cong(\mathbb{Z} / 4)^{2} \oplus \mathbb{Z} / 2 .
\end{gathered}
$$

We now have both the terms appearing in the splitting formula, and we conclude that the lower algebraic $K$-theory of the group $\Gamma$ is given by

$$
\mathrm{Wh}_{n}(\Gamma)= \begin{cases}\mathrm{Wh}(\Gamma) \cong \bigoplus_{\infty} \mathbb{Z} / 2, & n=1 \\ \widetilde{K}_{0}(\mathbb{Z} \Gamma) \cong(\mathbb{Z} / 4)^{2} \oplus \bigoplus_{\infty} \mathbb{Z} / 2, & n=0 \\ K_{-1}(\mathbb{Z} \Gamma) \cong \mathbb{Z}^{2}, & n=-1, \\ K_{n}(\mathbb{Z} \Gamma) \cong 0, & n \leq-1\end{cases}
$$

Looking up Table 7, one finds that these are precisely the values reported. 
Vol. 84 (2009) Lower algebraic $K$-theory of hyperbolic 3-simplex reflection groups

\section{References}

[Bar03] A. Bartels, On the domain of the assembly map in algebraic K-theory. Algebr. Geom. Topol. 3 (2003), 1037-1050. Zbl 1038.19001 MR 2012963

[Bas65] H. Bass, The Dirichlet unit theorem, induced characters, and Whitehead groups of finite groups. Topology 4 (1965), 391-410. Zbl 0166.02401 MR 0193120

[Bas68] H. Bass, Algebraic K-theory. W. A. Benjamin, New York 1968. Zbl 0174.30302 MR 0249491

[BFPP00] E. Berkove, F. T. Farrell, D. J. Pineda, and K. Pearson, The Farrell-Jones isomorphism conjecture for finite covolume hyperbolic actions and the algebraic $K$-theory of Bianchi groups. Trans. Amer. Math. Soc. 352 (2000), 5689-5702. Zbl 0954.19001 MR 1694279

[C80a] D. Carter, Lower K-theory of finite groups. Comm. Algebra 8 (20) (1980), 1927-1937. Zbl 0448.16017 MR 0590500

[C80b] D. Carter, Localization in lower algebraic K-theory. Comm. Algebra 8 (1980), 603-622. Zbl 0429.16019 MR 0561543

[CP02] F. X. Connolly and S. Prassidis, On the exponent of the cokernel of the forget-control map on $K_{0}$-groups. Fund. Math. 172(2002), 201-216.Zbl 0992.57022 MR 1898685

[CuR81] C. Curtis and I. Reiner, Methods of representation theory. Vol. I, Wiley, New York 1981. Zbl 0469.20001 MR 0632548

[CuR87] C. Curtis and I. Reiner, Methods of representation theory. Vol. II, Wiley, New York 1987. Zbl 0616.20001 MR 0892316

[D] J. Davis, Some remarks on Nil groups in algebraic $K$-theory. Preprint; arXiv:0803.1641v2.

[DKR] J. Davis, Q. Khan, and A. Ranicki, Algebraic $K$-theory over the infinite dihedral group. Preprint; arXiv:0803.1639v2.

[DQR] J. Davis, F. Quinn, and H. Reich, Algebraic $K$-Theory of virtually cyclic groups. In preparation.

[EH79] S. Endo and Y. Hironaka, Finite groups with trivial class group. J. Math. Soc. Japan 31 (1979), 161-174. Zbl 0396.20004 MR 0519042

[EM76] S. Endo and T. Miyata, On the projective class group of finite groups. Osaka J. Math. 13 (1976), 109-122. Zbl 0364.20009 MR 0409561

[EM80] S. Endo and T. Miyata, On the class group of dihedral groups. J. Algebra 63 (1980), 548-573. Zbl 0436.16006 MR 0570730

[FO] D. Farley and I. J. Ortiz, Three-dimensional crystallographic groups and algebraic $K$-theory. In preparation.

[F77] F. T. Farrell, The nonfiniteness of Nil. Proc. Amer. Math. Soc. 65 (1977), 215-216. Zbl 0365.18021 MR 0450328

[FJ93] F. T. Farrell and L. Jones, Isomorphism conjectures in algebraic $K$-theory. J. Amer. Math. Soc. 6 (1993), 249-297. Zbl 0798.57018 MR 1179537

[FJ95] F. T. Farrell and L. Jones, The lower algebraic $K$-theory of virtually infinite cyclic groups. K-theory 9 (1995), 13-30. Zbl 0829.19002 MR 1340838 
[G07] J. Grunewald, Non-finiteness results for nil-groups. Algebr. Geom. Topol. 7 (2007), 1979-1986. Zbl 1127.19004 MR 2366184

[Ha87] D. R. Harmon, $N K_{1}$ of finite groups. Proc. Amer. Math. Soc. 100 (1987), 229-232. Zbl 0625.18003 MR 0884456

[JKRT99] N. W. Johnson, J. G. Ratcliffe, R. Kellerhals, and S. T. Tschantz, The size of a hyperbolic Coxeter simplex. Transform. Groups 4 (1999), 329-353. Zbl 0953.20041 MR 1726696

[JKRT02] N. W. Johnson, J. G. Ratcliffe, R. Kellerhals, and S. T. Tschantz, Commensurability classes of hyperbolic Coxeter groups. Linear Algebra Appl. 345 (2002), 119-147. Zbl 1033.20037 MR 1883270

[LO07] J.-F. Lafont and I. J. Ortiz, Relative hyperbolicity, classifying spaces, and lower algebraic K-theory. Topology 46 (2007), 527-553. Zbl 1132.19001 MR 2363244

[LO08] J.-F. Lafont and I. J. Ortiz, Relating the Farrell Nil-groups to the Waldhausen Nilgroups. Forum Math. 20 (2008), 445-455. Zbl 1147.19005 MR 2418200

[LMO] J.-F. Lafont, B. Magurn, and I. J. Ortiz, Lower algebraic $K$-theory of certain reflection groups. In preparation.

[LW] W. Lück and M. Weiermann, On the classifying space of the family of virtually cyclic subgroups. Pure Appl. Math. Q., to appear.

[Ma78] B. Magurn, $S K_{1}$ of dihedral groups. J. Algebra 51 (1978), 399-415. Zbl 0376.16026 MR 0498804

[Ma80] B. Magurn, Whitehead groups of some hyperelementary groups. J. London Math. Soc. (2) 21 (1980), 176-188. Zbl 0418.16015 MR 0576195

[Ma06] B. Magurn, Explicit $K_{1}$ of some modular groups rings. J. Pure Appl. Algebra 206 (2006), 3-20. Zbl 1093.19001 MR 2220078

[Ma07] B. Magurn, Explicit $K_{2}$ of some finite groups rings. J. Pure Appl. Algebra 209 (2007), 801-811. Zbl 1120.19002 MR 2298858

[Mi66] J. Milnor, Whitehead torsion. Bull. Amer. Soc. 72 (1966), 358-426. Zbl 0147.23104 MR 0196736

[O89] R. Oliver, Whitehead groups of finite groups. London Math. Soc. Lecture Notes Ser. 132, Cambridge University Press, Cambridge 1989. Zbl 0636.18001 MR 0933091

[Or04] I. J. Ortiz, The lower algebraic $K$-theory of $\Gamma_{3}$. K-theory 32 (2004), 331-355. Zbl 1068.19004 MR 2112901

[Pe98] K. Pearson, Algebraic $K$-theory of two dimensional crystallographic groups. $K$ theory 14 (1998), 265-280. Zbl 0907.19001 MR 1633509

[Qu82] F. Quinn, Ends of maps II. Invent. Math. 68 (1982), 353-424. Zbl 0533.57008 MR 0669423

[Ra07] R. Ramos, Non finiteness of twisted Nils. Bol. Soc. Mat. Mexicana (3) 13 (2007), 55-64. MR 2468022

[Re76] I. Reiner, Class groups and Picard groups of group rings and orders. Conference Board of the Mathematical Sciences, Regional Conference Ser. Math. 26, Amer. Math. Soc., Providence, RI, 1976. Zbl 0326.16025 MR 0404410 
[RU74] I. Reiner, and S. Ullom, Remarks on class groups of integral group rings. Symposia Math. XIII (Convegno di Gruppi e loro Rappresentazioni, INDAM, Rome, 1972), Academic Press, London 1974, 501-516. Zbl 0305.20007 MR 0367043

[Se77] J. P. Serre, Linear representations of finite groups Grad. Texts in Math. 42, SpringerVerlag, New York 1977. Zbl 0355.20006 MR 0450380

[Sw60] R. G. Swan, Induced representations and projective modules. Ann. of Math. (2) 71 (1960), 552-578. Zbl 0104.25102 MR 0138688

[V67] E. B. Vinberg, Discrete groups generated by reflections in Lobachevskii spaces. Math. USSR-Sb. 1 (1967), 429-444. Zbl 0166.16303 MR 0207853

[Wd78] F. Waldhausen, Algebraic K-theory of generalized free product I, II. Ann. of Math. 108 (1978), 135-256. Zbl 0397.18012 MR 0498807

[W74] C. T. C. Wall, Norms of units in group rings. Proc. London Math. Soc. 29 (1974), 593-632. Zbl 0302.16013 MR 0376746

[We] C. Weibel, $N K_{0}$ and $N K_{1}$ of the groups $C_{4}$ and $D_{4}$. Comment. Math. Helv. 84 (2009), 339-349.

Received May 6, 2007

Jean-François Lafont, Department of Mathematics, Ohio State University, Columbus, OH 43210, U.S.A.

E-mail: jlafont@math.ohio-state.edu

Ivonne J. Ortiz, Department of Mathematics and Statistics, Miami University, Oxford, OH 45056, U.S.A.

E-mail: ortizi@muohio.edu 